\title{
A comparative study on effect of plain- and wavy-wall confinement on wake characteristics of flow past circular cylinder
}

\author{
R DEEPAKKUMAR $^{1}, \mathrm{~S} \mathrm{JAYAVEL}^{1, *}$ and SHALIGRAM TIWARI ${ }^{2}$ \\ ${ }^{1}$ Indian Institute of Information Technology Design \& Manufacturing Kancheepuram, Chennai 600127, India \\ ${ }^{2}$ Indian Institute of Technology Madras, Chennai 600036, India \\ e-mail:sjv@iiitdm.ac.in
}

MS received 19 August 2016; revised 7 December 2016; accepted 14 December 2016

\begin{abstract}
A first attempt is made for identifying the wake characteristics of circular cylinder confined by a wavy wall at laminar flow regime. Numerical study of flow characteristics past circular cylinder with wavy-wall confinement perpendicular to cylinder axis has been carried out in the range of Reynolds number 20-100. The finite volume-based CFD solver Ansys Fluent (Version 15.0) is used for computations. The results are presented in the form of streamline plots, mean drag co-efficient, flow separation angle and recirculation length. Wavywall confinement leads to highly significant changes in the cylinder wake such as the evolution of strong $x$-plane vortices, enhanced fluid mixing, wake suppression near the crest region and vortex stretching near the trough region on the downstream of the cylinder has been observed. Flow separation angle varies significantly along the axis of the cylinder. Increased wall shear stress on rear surface of the cylinder has also been observed. The part of vorticity magnitude as compared to strain rate has been distinguished and identified using vortex identification methods such as Q-criterion and Lambda-2 criterion.
\end{abstract}

Keywords. Bluff body; wake; flow separation; wavy-wall confinement; CFD simulation; finite volume method.

\section{Introduction}

The flow across cross-confined circular cylinder plays a significant role in many engineering systems such as wind structure interaction of civil engineering industries, tube banks of heat exchanger in chemical industries and food processing and textile industries. It is well known that the effect of confining walls on flow characteristics is significant. On the basis of orientation of cylinder axis with respect to wall confinement, the available literature can be broadly grouped into two categories, namely, cylinder axis parallel to the confining walls and cylinder axis perpendicular to the confining walls. The first category can be effectively studied using two-dimensional computational domain with an assumption that the cylinder is long; however, the latter leads to complex flow characteristics due to cylinder and wall interaction and requires threedimensional computational domain. Instead of plain walls, if the walls are of wavy geometry then the flow characteristics in presence of built-in circular cylinders are highly complex. Experimental investigations on steady and unsteady confined flow past circular cylinder with aspect ratio $\alpha=30$ and blockage ratio $\beta=1 / 3$ were conducted by Rehimi et al [1] and the results were compared with

*For correspondence unconfined flow. The von Karman instability, which occurs at $\operatorname{Re}=47$ for unconfined flow, was delayed due to the confinement and found to occur at $\operatorname{Re}=108$. A detailed study on three-dimensional wake transition regime for flow past circular cylinder was conducted by Williamson $[2,3]$ and characterised the wake transition regime by two distinct three-dimensional modes of instability based on the wavelength. The delay in vortex shedding due to wall confinement was observed and reported in the numerical study due to Tiwari et al [4]. A complete suppression of vortex shedding is observed by the present author Deepakkumar et al [5] at Reynolds number 200, in presence of local waviness in the confining walls. Sahin et al [6] have studied the $\beta$ effect on vortex shedding characteristics and observed that the increasing value of $\beta$ leads to re-stabilised steady flow. Two- and three-dimensional studies of flow around circular cylinder were conducted by Nicolas et al [7]. They have reported that the two-dimensional flow assumption is valid for $\operatorname{Re} \leq 180$ for unconfined flow. Moreover, Williamson [2] has also reported that the transition to three-dimensionality occurs in the range $180 \leq \mathrm{Re}$ $\leq 194$. On the other hand, Nicolas et al [7] have reported that transition to three-dimensionality was delayed (up to $\mathrm{Re}=210)$ due to confinement. Zovatto et al [8] have conducted a numerical study of steady flow past circular cylinder confined between channel walls, and the study was 
extended up to the critical Reynolds number corresponding to the physical appearance of three-dimensional instabilities. As the cylinder is near to one of the walls, the transition was delayed due to interaction of wake with wall boundary layer. So, the cylinder with wall confinement significantly affects the wake and vortex shedding characteristics for the corresponding Reynolds number.

Study of confinement along the axis of the cylinder (aspect ratio, $\alpha=L / D$ ) was also reported in literature. Wake characteristics in terms of Strouhal number, St, was studied by Norberg [9]. The influence of $\alpha$ on St was found negligible for $\alpha>40$. The experimental work from Gerich and Eckelmann [10] reveals that the delay in shedding occurs when a plate is fixed at one end of the cylinder, thus resulting in shifting of critical Reynolds number to a higher value. Similarly, in a recent study, Huang et al [11] have observed the effect of end wall on vortex shedding at $\alpha=6, \beta=0.1$. In their numerical simulation, they have noticed that two-dimensional results can be reproduced only with free-slip end condition; on the other hand, if noslip condition is imposed, the vortex shedding is suppressed near to the boundary and the shedding pattern was substantially affected near the mid-plane of the cylinder. Attached flow due to confinement on cylinder surface results in increasing frictional drag [12] and leads to delay in separation angle and in the critical Reynolds number associated with vortex shedding [1, 6, 8]. Bearman [13] discussed the effects of introducing wavy base, where large changes in vortex shedding and wake structure were reported. The effect of fin waviness and fin spacing on the lateral vortex structure has been investigated by Zhang et al [14]. They found that for small separation the viscous force dominates and dampens the lateral vortices induced by wavy-wall curvature. This leads to a streamlined and fully developed flow.

Thus, literature is rich in the study of flow behavior past circular cylinder with plain-wall confinement, whereas, to the best knowledge of the authors, the study of flow characteristics with wavy-wall cross confinement is limited. In particular, this is the first attempt for identifying the wake characteristics of cylinder confined by wavy walls. In the present work, three-dimensional computational domain has been used to study the significant changes in the cylinder wake flow characteristics due to wavy-wall confinement and the results are compared with plain-wall confinement.

\section{Problem statement}

Three-dimensional numerical and experimental studies for flow past confined cylinder were carried out by Riberio et al [15] for $\beta=1 / 2$ and $\alpha=16,8$ and 2 . The current problem is described as three-dimensional steady incompressible flow past circular cylinder confined with wavy walls as shown in figure 1. All length scales are non-dimensionalised with respect to the characteristic length, $D$, where $D$ is the cylinder diameter. Riberio et al [15] have reported that the upstream influence of cylinder was observed up to $(x / D) \approx$ -2 , where $x=0$ is the cylinder centre. To take this phenomenon into account and to minimise the inlet boundary effect, the computational domain inlet has been located at $6 \mathrm{D}$ from the cylinder centre as shown in figure 2. Moreover, the main interest of the present work is to identify the wake characteristics due to wavy-wall confinement. Therefore, the influence due to flow development has been precluded by imposing a fully developed inlet flow boundary condition. The length of waviness in the downstream, measured from the cylinder axis, is $5.5 \mathrm{D}$. The length has been chosen based on the recirculation length $\left(\mathrm{L}_{\mathrm{r}}\right.$ $\approx 5.5 \mathrm{D}$ ) due to unconfined flow past circular cylinder, as reported by Deepakkumar and Jayavel [16], Rajani et al [17], etc. The effects of exit boundary condition on upstream flow characteristics are closely studied by varying the downstream length. Based on the aforementioned study, the outlet boundary is imposed at 24D from the cylinder centre. The confinement, that is, the distance between the two walls, $\mathrm{L}=2 \mathrm{D}$ and the amplitude of waviness, $\mathrm{A}=0.15 \mathrm{D}$, with wave length $\lambda=2 \mathrm{D}$ are considered based on the greater impact of practical relevance, such as fin and tube heat exchanger. The cylinder is placed symmetrically with respect to transverse $y$-direction. The study has been

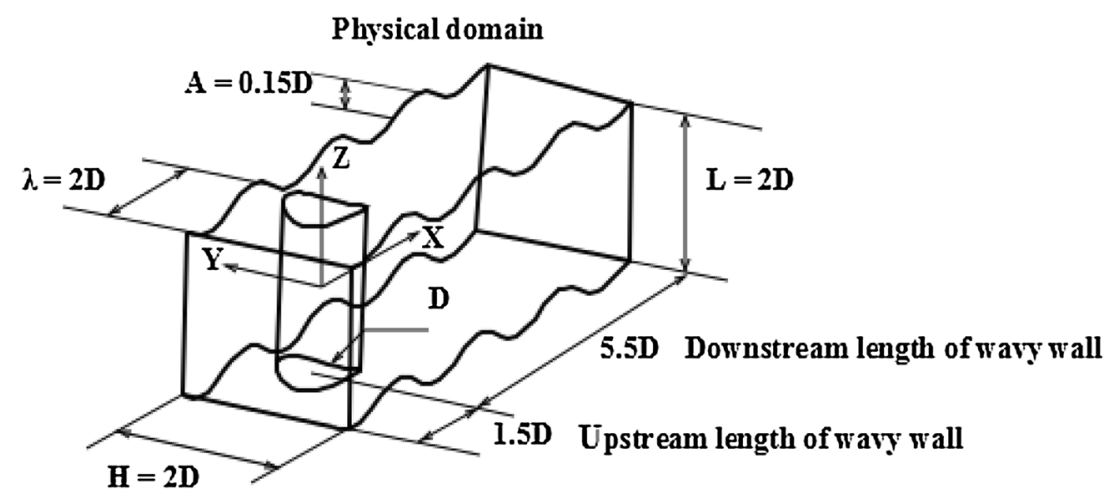

Figure 1. Schematic representation of the physical domain. 


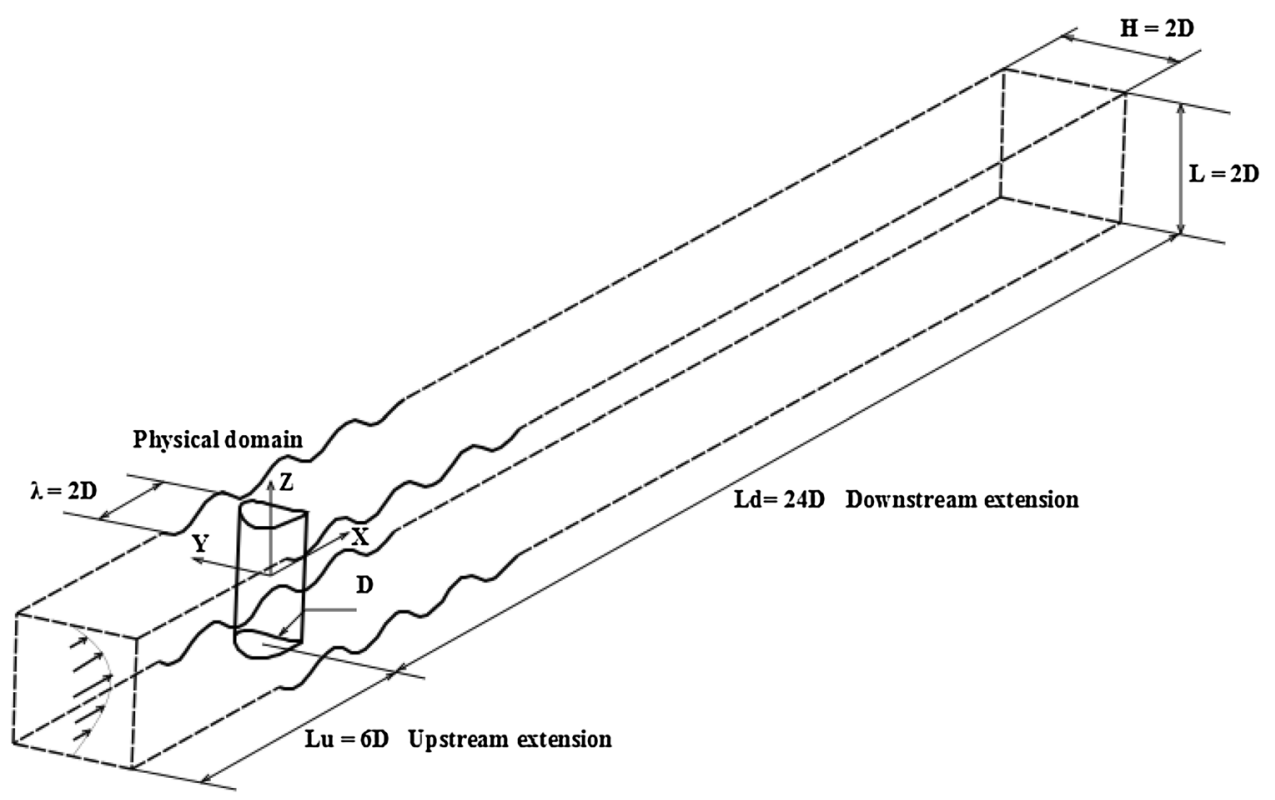

Figure 2. Computational domain with upstream and downstream extensions.

conducted for various Reynolds number in the range 20 to 100 , with air as the working fluid.

\section{Governing equations and solution procedure}

The continuity and Navier-Stokes equations for the laminar flow in a domain of volume $\mathrm{V}$ bounded by a closed surface $\mathrm{S}$ is expressed in the following general convection-diffusion-source integral form (Eqs. 1 and 2). Where $d \vec{S}$ is the differential area vector of the surface directed along normal at the given point on its surface. Eq. (1) is the mass conservation equation, which can be deduced from the general equation given in Eq. (2).

$$
\begin{gathered}
\frac{\partial}{\partial t} \int_{V} \rho d V+\int_{S} \rho \vec{u} \cdot d \vec{S}=0 \\
\frac{\partial}{\partial t} \int_{V} \rho \phi d V+\int_{S}[\rho \vec{u} \phi] \cdot d \vec{S}=\int_{S}\left[\Gamma_{\phi} \nabla \phi\right] \cdot d \vec{S}+\int_{V} S_{\phi} d V
\end{gathered}
$$

where $\varphi, \Gamma$ and $S$ are, respectively, the general transport variable, diffusion coefficient and source term. The equations for mass and momentum conservation are obtained by

Table 1. Variables for general transport equation.

\begin{tabular}{lccc}
\hline Equation & $\phi$ & $\Gamma_{\phi}$ & $S_{\phi}$ \\
\hline Continuity & 1 & 0 & 0 \\
Momentum & $\mathrm{u}, \mathrm{v}, \mathrm{w}$ & $\mu$ & $\frac{\partial p}{\partial x}, \frac{\partial p}{\partial y}, \frac{\partial p}{\partial z}$
\end{tabular}

Table 2. Summary of boundary conditions.

\begin{tabular}{lc}
\hline Boundary & Condition \\
\hline Inlet & $u=U_{\max }\left[1-\frac{z^{2}}{(L / 2)^{2}}\right], \quad v=0, w=0$ \\
Exit & $\frac{\partial u}{\partial x}=0, \frac{\partial v}{\partial x}=0, \frac{\partial w}{\partial x}=0, \frac{\partial p}{\partial x}=0$ \\
$\begin{array}{l}\text { Sides: front and rear } \\
\text { surfaces }\end{array}$ & $\frac{\partial u}{\partial y}=0, v=0, \frac{\partial w}{\partial y}=0, \frac{\partial p}{\partial y}=0$ \\
$\begin{array}{l}\text { Walls: cylinder, top and } \\
\text { bottom }\end{array}$ & $u=v=w=0$ \\
\hline
\end{tabular}

assigning appropriate values to the variable $\varphi, \Gamma$ and $S$ as presented in table 1.

The boundaries of the computational domain fall under four categories, namely, inlet, exit, symmetrical side walls and no-slip solid surfaces. The boundary conditions are summarised in table 2. Fully developed flow at the inlet is imposed by using UDF. Flow is assumed to be free from velocity gradients normal to the exit plane as it is located sufficiently away from the cylinder. No-slip boundary condition has been imposed at the cylinder top and bottom walls. The front and rear surfaces are imposed with freeslip boundary condition to minimise boundary effect on wake characteristics and the distance $(\mathrm{H}=2 \mathrm{D})$ is considered with a single tube isolated from a bank of tubes. Finite volume- based CFD solver Ansys Fluent (Version 15.0) is used for solving the governing equations with the boundary conditions as listed in table 2. SIMPLE is the numerical algorithm employed in the present study to solve steadystate pressure-based coupled equations. The gradients of solution variables at cell centers are determined using leastsquare cell-based approach. Cell-face pressures are calculated using second-order interpolation scheme. As the grids 


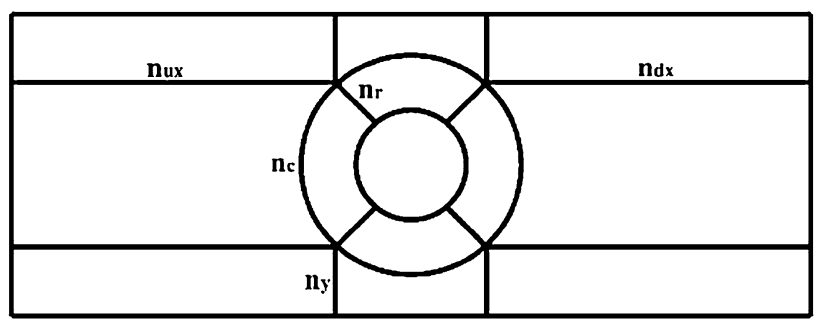

Figure 3. Schematic representation of various blocks with notations indicating the number of grids.

are aligned with the flow direction, to obtain the better accuracy, the convective terms are discretised by higherorder QUICK scheme. The convergence criterion for the residuals is taken as $1 \times 10^{-6}$.

\section{Grid generation}

The computational domain shown in figure 2 has been discretised as a structured grid using Ansys ICEM CFD meshing tool. Block-structured grid-generation methodology is employed and the fluid domain has been divided into 12 blocks to generate high-quality grid. The $O$-grid block is created around the cylinder for generating very fine grids. The grids are clustered around the cylinder and also near the top and bottom walls to capture the steep gradients in flow field near the solid walls. A minimum grid size of $0.001 D$ is maintained near the cylinder surface. The grid size adjacent to the top and bottom walls is maintained as $0.01 D$. Figure 3 shows the notations used to indicate the number of grid nodes in each block. A two-dimensional representation of the three-dimensional grid is shown in figure 4 along the planes passing through the origin.

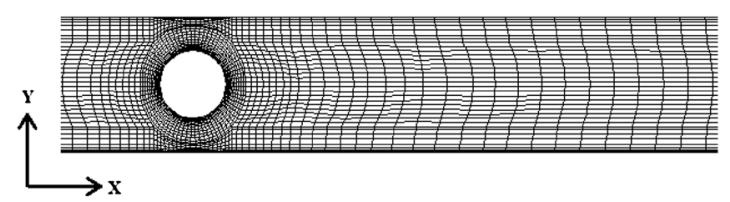

(a)

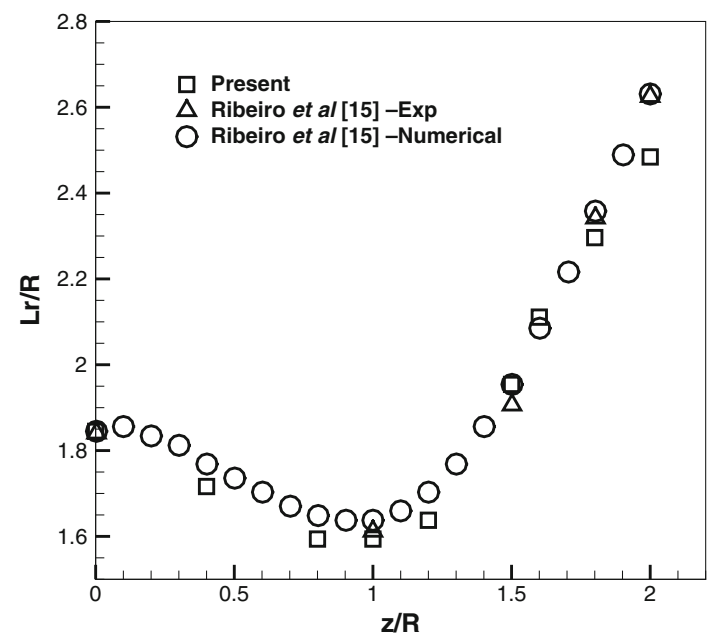

Figure 5. Variation of recirculation length along z-direction in $y=0$ plane at $\operatorname{Re}=26.6$.

\section{Results and discussion}

The main objective of the present work is to identify the wake flow characteristics of the circular cylinder confined by a wavy wall in the lower range of Reynolds number. The results are compared with the plain-wall-confined cylinder. The present computational methodology has been validated using those available in literature [15]. Initially, a preliminary study has been carried out for flow between plain and wavy confining walls without cylinder in order to study the significance of wavy-wall confinement on the flow characteristics. Interesting flow characteristics due to wavy-wall confinement has been observed. In order to identify the significance of wavy-wall confinement on free stream region, centreline velocity has been monitored. It is noticed that the free stream flow is accelerated due to the projection
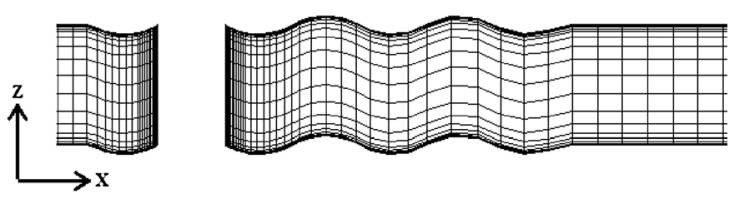

(b)

Figure 4. Representation of the structured mesh. (a) Near the cylinder and (b) confined region between the wavy walls.

Table 3. Number of nodes in various blocks for different grids.

\begin{tabular}{|c|c|c|c|c|c|c|c|c|c|c|}
\hline Grid & $n_{r}$ & $n_{c}$ & $n_{y}$ & $n_{u x}$ & $n_{d x}$ & $n_{z}$ & $\mathrm{C}_{\mathrm{D}}$ & $\mathrm{L}_{\mathrm{r}}(\mathrm{mm})$ & Variation in $\mathrm{C}_{\mathrm{D}}(\%)$ & Variation in $\mathrm{L}_{\mathrm{r}}(\%)$ \\
\hline$G_{1}$ & 20 & 20 & 10 & 20 & 40 & 20 & 5.5184 & 11.1787 & & \\
\hline$G_{2}$ & 24 & 24 & 12 & 24 & 48 & 24 & 5.5458 & 10.2149 & 0.50 & 8.62 \\
\hline$G_{3}$ & 28 & 28 & 14 & 28 & 56 & 28 & 5.5520 & 10.4318 & 0.11 & 2.12 \\
\hline$G_{4}$ & 40 & 30 & 20 & 30 & 60 & 30 & 5.5545 & 10.7885 & 0.04 & 3.42 \\
\hline$G_{5}$ & 40 & 36 & 24 & 36 & 72 & 36 & 5.5559 & 10.7899 & 0.03 & 0.01 \\
\hline$G_{4 a}$ & 40 & 30 & 20 & 30 & 60 & 45 & 5.5593 & 10.8337 & 0.09 & 0.42 \\
\hline
\end{tabular}



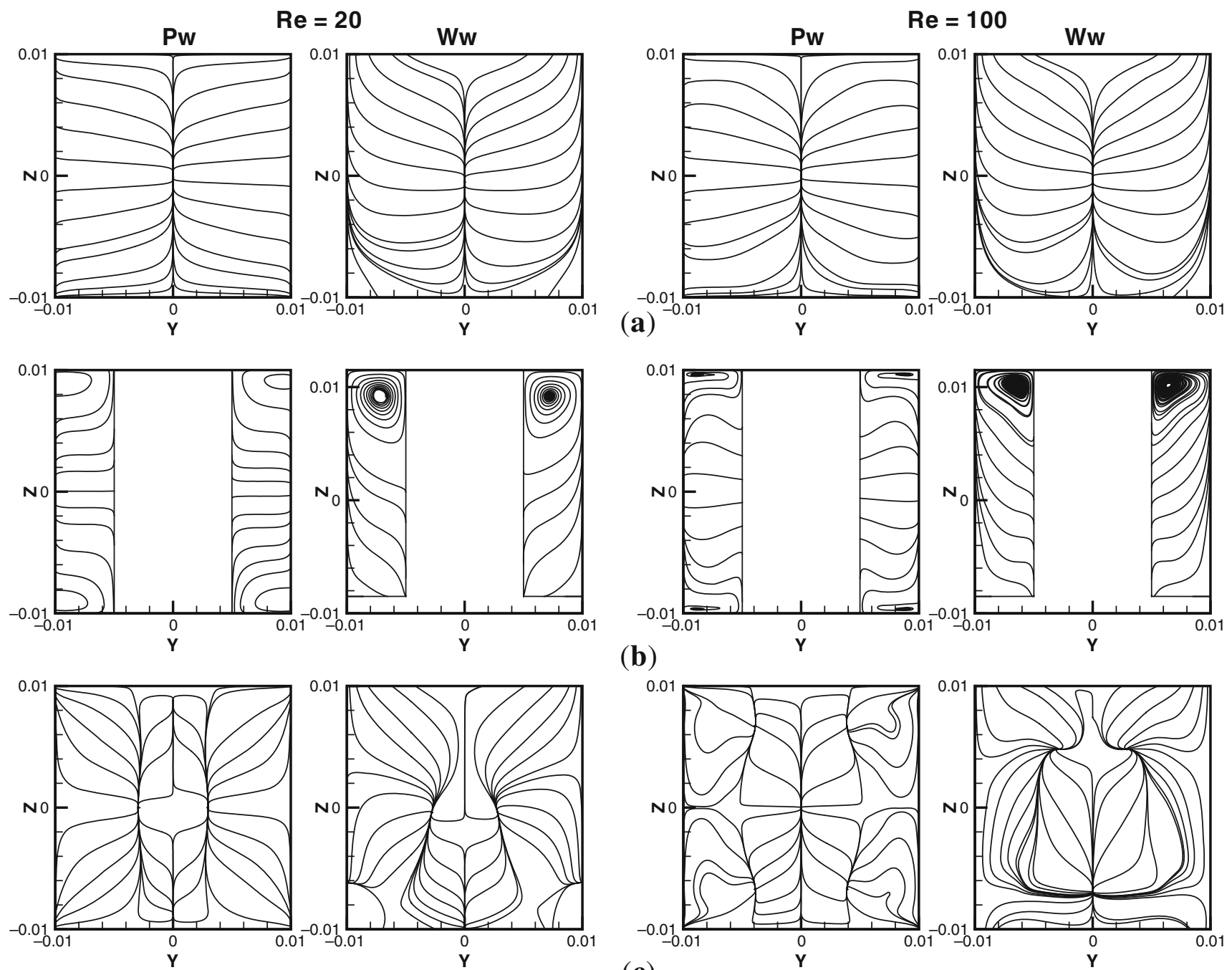

(b)
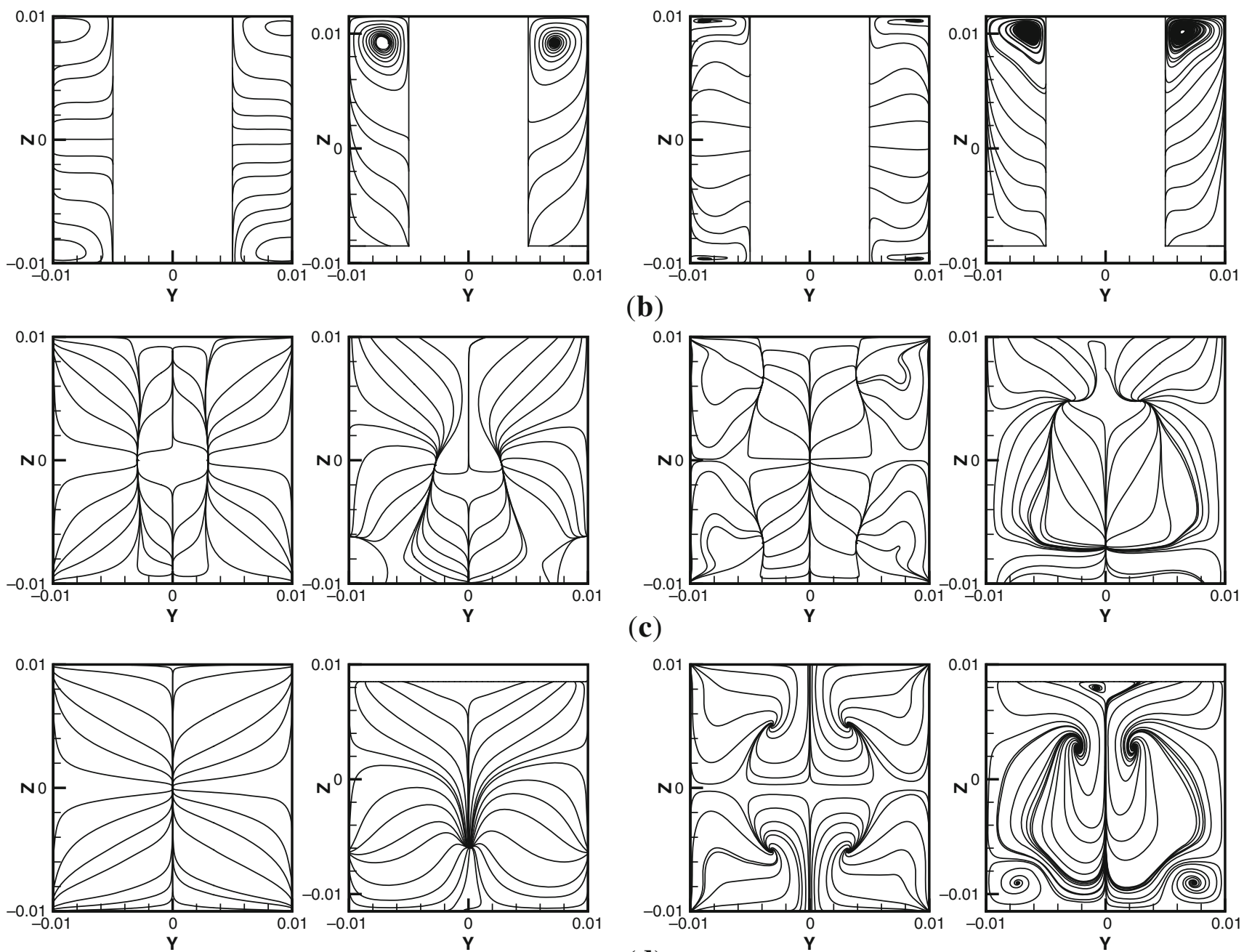

(c)
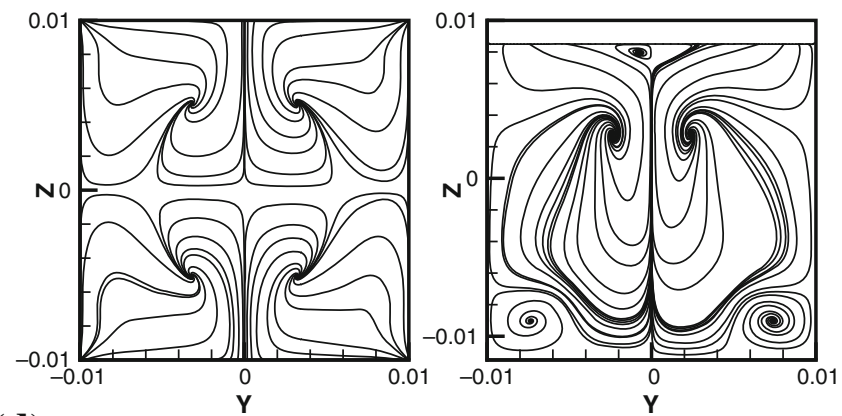

(d)

Figure 6. Streamline plots at various $x$-planes near the cylinder. (a) $x=-0.5 \mathrm{D}$, (b) $x=0,(\mathbf{c}) x=0.5 \mathrm{D}$ and (d) $x=1 \mathrm{D}$.

of crest region of the wavy wall into the main stream flow. The detailed results are omitted due to space constraints. Hence, the following section includes a detailed grid independence study (section 5.1), validation of the computational methodology (section 5.2), qualitative and quantitative flow characteristics (sections 5.3 and 5.4) and vortex identification in section 5.5.

\subsection{Grid independence study}

Thorough grid independence study has been conducted using different number of grids along $x$-, $y$ - and $z$-directions as presented in table 3 . To capture steep gradients near the boundary layer, refinement is done near the solid surfaces. With increasing Reynolds number, the number of grids required also increases [18]. Therefore, grid independence 


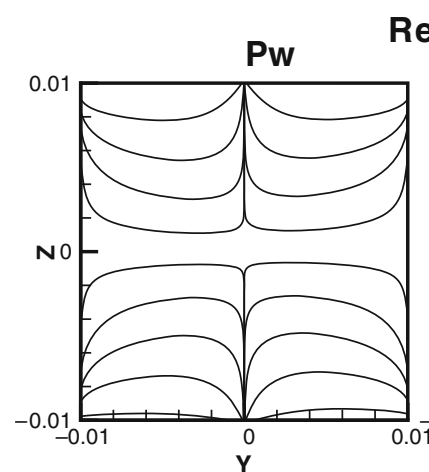

$\operatorname{Re}=20$
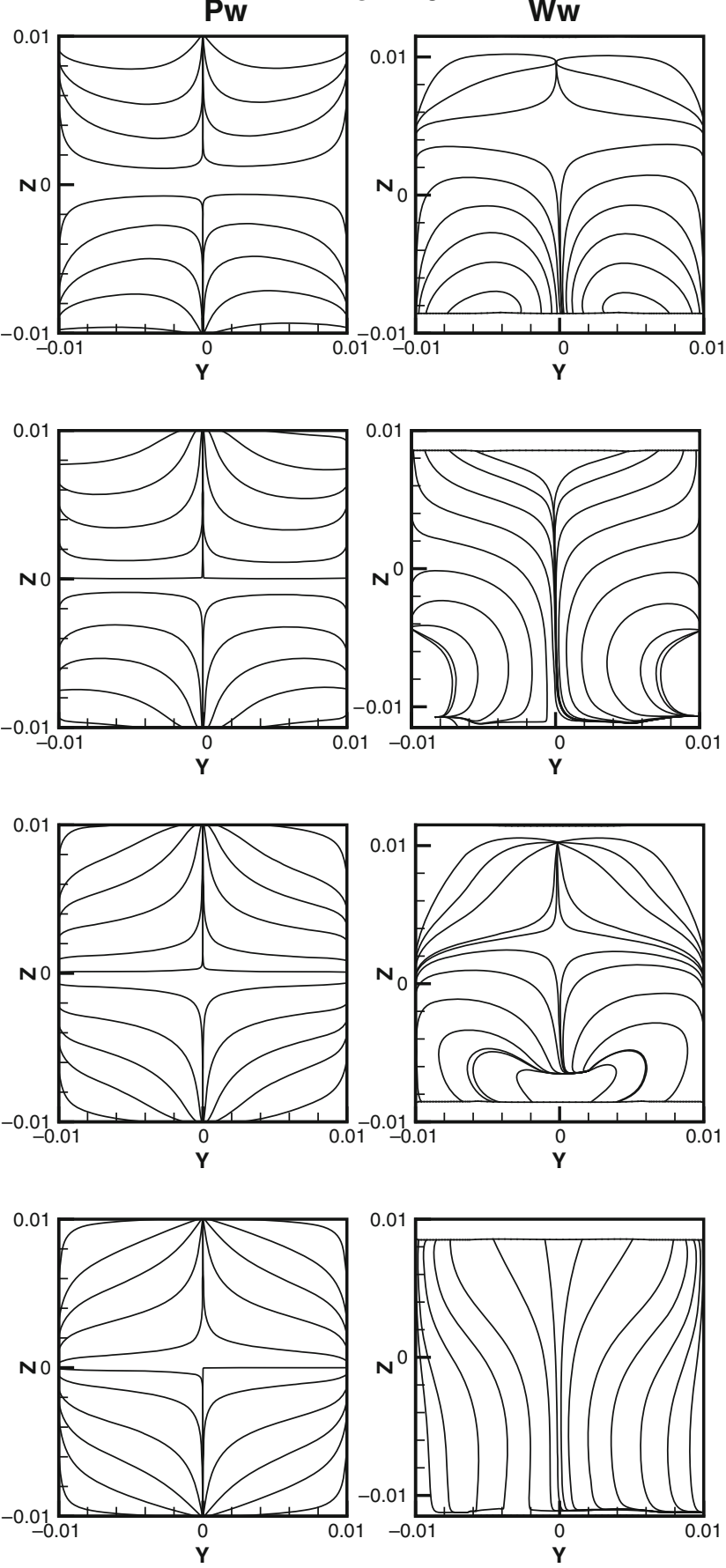

(a)
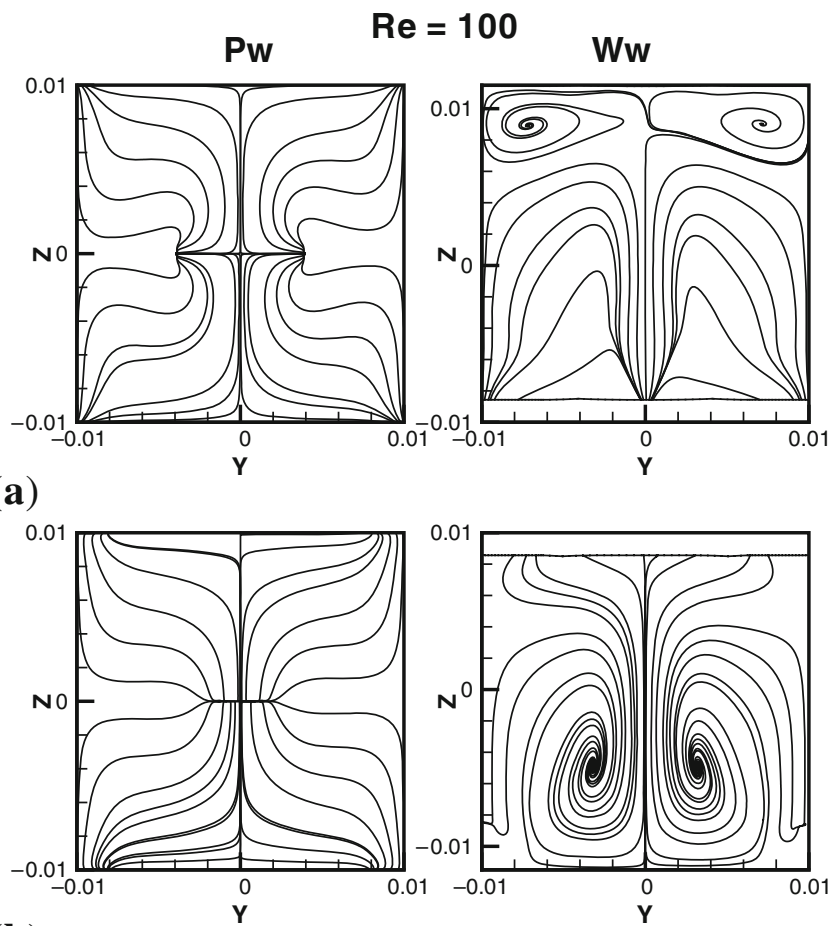

(b)
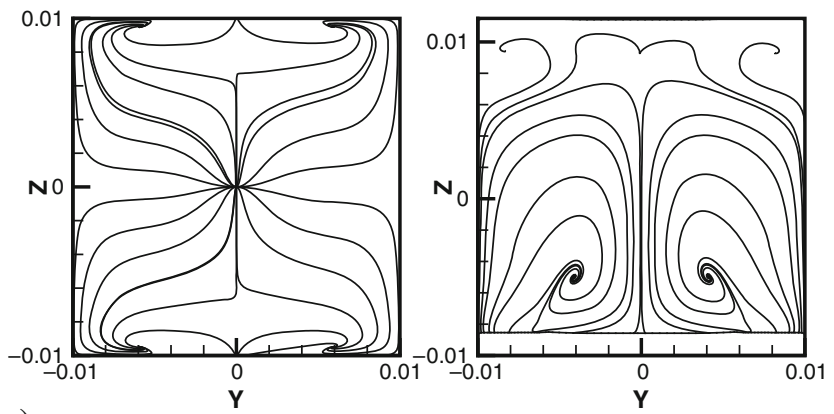

(c)
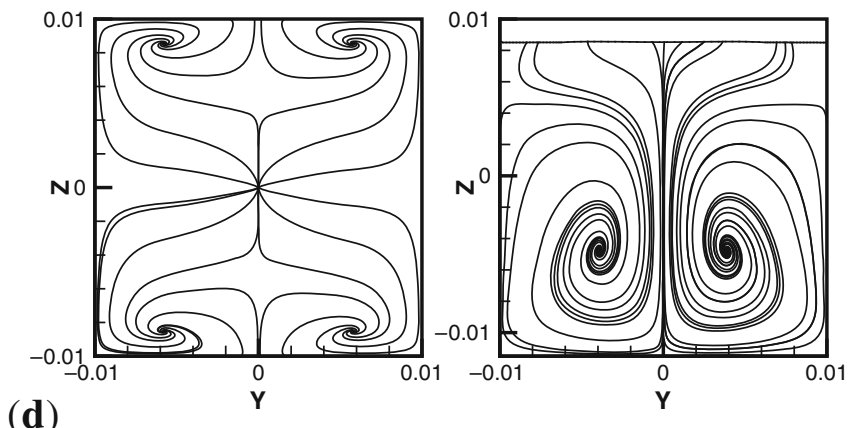

Figure 7. Streamline plots at various $x$-planes in the downstream. (a) $x=2 \mathrm{D},(\mathbf{b}) x=3 \mathrm{D}$, (c) $x=4 \mathrm{D}$ and (d) $x=5 \mathrm{D}$.

test has been conducted for various Reynolds number $\left(\operatorname{Re}=20,40,60,80\right.$ and 100), the variations in $C_{D}$ and $L_{r}$ due to change in grid size are monitored and tabulated in table 3 for the Reynolds number 100 . Arbitrarily grid $G_{1}$ is taken and its details are listed in table 3 . The number of grids as designated by $\mathrm{G}_{2}$ and $\mathrm{G}_{3}$ are, respectively, 1.2, 1.4 times of $G_{1}$ the number of grids in $G_{5}$ is 1.2 times of $G_{4}$ with further refinement near the cylinder. Comparison of results obtained using $\mathrm{G}_{4}$ and $\mathrm{G}_{5}$ show the percentage change in the results are 0.284 and 0.01 , respectively, for $C_{D}$ and $L_{r}$. Further grid refinement along $z$-direction has also been made to accurately capture the wavy-wall effect and the grids used are designated as $\mathrm{G}_{4 \mathrm{a}}$ and $\mathrm{G}_{4 \mathrm{~b}}$. The percentage change in the results is within $1 \%$. Therefore, $\mathrm{G}_{4 \mathrm{a}}$ is identified as optimum grid and is used for further computations. 


\subsection{Validation of the computed results}

The present computational results are validated using the numerical and experimental results of Ribeiro et al [15]. Their results correspond to the study of three-dimensional effects on steady flow past confined circular cylinder located in a rectangular channel. A computational domain similar to the one used by Ribeiro et al [15] with an aspect ratio $\alpha=2$ and blockage ratio $\beta=0.5$ is used for validating the present computational methodology. The validations include recirculation length along $z$-direction, normalised $x$-component velocity along $x$ - and $y$-directions. The present results are found to be in good agreement with those available in Ribeiro et al [15]. The variation of recirculation length along $z$-direction for $\mathrm{Re}=26.6$ is shown in figure 5 .

The flow is symmetric along $y$-direction; therefore, $y=0$ plane is taken for calculating the recirculation length. The recirculation length is large near the confining walls than at the mid-plane. This is due to the wall interaction with the flow leading to higher shearing effect [15]. The computed recirculation lengths are in good agreement with literature results and the maximum deviation is $2.5 \%$. The distance measured along the centreline of the flow domain (i.e., at $y=z=0$ line) between rear stagnation point and the point where the change in direction of the $x$-component velocity is noticed gives the recirculation length.

\subsection{Effect of wavy-wall confinement on qualitative flow characteristics}

The curvature of wavy-wall confinement leads to the generation of $x$-plane vortices as shown in figures 6 and 7 . However, with plain-wall confinement, there are no such vortices up to the cylinder wake, thereafter, there exists near to top and bottom walls and the streamlines are symmetric with respect to $y$ and $z$ directions. At low Reynolds number $(\operatorname{Re}=20)$, the $x$-plane vortices are observed only near the cylinder and curvature interaction as shown in figure $6 \mathrm{~b}$. On the other hand, at higher Reynolds number $(\operatorname{Re}=100)$, the $x$-plane vortices are observed near all the curvatures of wavy-wall confinement as shown in figure 7 . In the downstream, away from the cylinder, the shape of these vortices closely resembles the human lungs. Such three-dimensionality in the flow will lead to better advection by disrupting the wall boundary layer, which is a favorable phenomenon for effective flow mixing and heat transfer enhancement.

It is well known that for flow past unconfined circular cylinder, at $\operatorname{Re}=100$, the flow is two-dimensional. The streamline plots in transverse $y$-plane for $\operatorname{Re}=20$ and 100 are shown in figures 8 and 9, respectively. In the planes at $y= \pm 0.25 D$, a pair of symmetric vortices are observed at $\mathrm{Re}=100$ with plain-wall confinement. On the other hand, unsymmetrical vortices are observed with wavy-wall confinement. The presence of these vortices clearly indicates the three-dimensional flow behavior due to cross confinement. Moreover, these vortices are steady-standing vortices. In the case of wavy wall, although the flow is laminar, symmetry is present only in transverse $y$-direction and not in $z$-direction due to alternative wavy nature of the confining walls. The mid-y-plane streamline plot in figures $8 \mathrm{~d}$ and $9 \mathrm{~d}$ shows the boundary of recirculation region. Moreover, there is no significant effect of cylinder in the transverse direction beyond $y= \pm 0.75 \mathrm{D}$.

The streamline plots as shown in figures 10 and 11 indicate the size of the vorticity at various $z$-planes. Mid- $z$ plane vortices as shown in figures $10 \mathrm{~d}$ and $11 \mathrm{~d}$ are not much influenced by the wall effect. Wavy-wall confinement leads to the suppression of the $z$-plane vortices near the crest region $(z=0.75 \mathrm{D})$, whereas the trough region enhances the development of the vortices $(z=-0.75 \mathrm{D})$. Although the vortices are observed only near the bottom wall of the wavy-wall confinement as shown in figure $10 \mathrm{~g}$, the vortex size is slightly larger for wavy-wall confinement compared to the plain-wall confinement. This is due to the immediate trough region of the wavy-wall, which stretches the already formed vortex, thus recirculation length increases. In the wake region, fluid near the confining walls is redirected towards the mid-plane as shown in figure $10 \mathrm{f}$ and g. This is clearly visible with plain wall and is in accordance with figure 9 , confirming three-dimensionality in the wake region. Unlike plain-wall-confined cylinder, the vortices in the $z$-planes are asymmetrical in the presence of wavy-wall-confined cylinder (figure10a to g) due to the alternate nature of the crest and valley at the cylinder end. This asymmetrical nature was also observed at Reynolds number 100 (figure 11a to $\mathrm{g}$ ). This is due to the dissimilar confinement of the cylinder along its axis, which completely suppresses the vortex formation near to the top end of the cylinder where the presence of a crest in the immediate downstream of the cylinder diverts the near wall fluid towards the bottom end of the cylinder. The presence of the valley at the bottom end of the cylinder also accelerates the flow diversion towards the bottom of the wake region.

Recirculation region in the wake of both plain- and wavy-wall confinements are clearly identified using isosurfaces of $u=0$ as shown in figure 12. The effect of confining wavy wall on the recirculation region is clearly visible from figure $12 \mathrm{~b}$ and $\mathrm{d}$. The curvature in the wavy wall redirects the flow and reduces the recirculation region.

\subsection{Effect of wavy-wall confinement on quantitative flow characteristics}

Effect of Reynolds number on various flow characteristics such as mean drag co-efficient $\left(\mathrm{C}_{\mathrm{D}}\right)$, recirculation length $\left(\mathrm{L}_{\mathrm{r}}\right)$ and separation angle $\left(\theta_{\mathrm{s}}\right)$ are shown in figure. 13. The values of $L_{r}$ and $\theta_{s}$ are taken at the mid-z-plane for 
(a) $x=-0.5 \mathrm{D}$ (b) $\mathrm{x}=0$ (c) $\mathrm{x}=0.5 \mathrm{D}$ (d) $\mathrm{x}=1 \mathrm{D}$
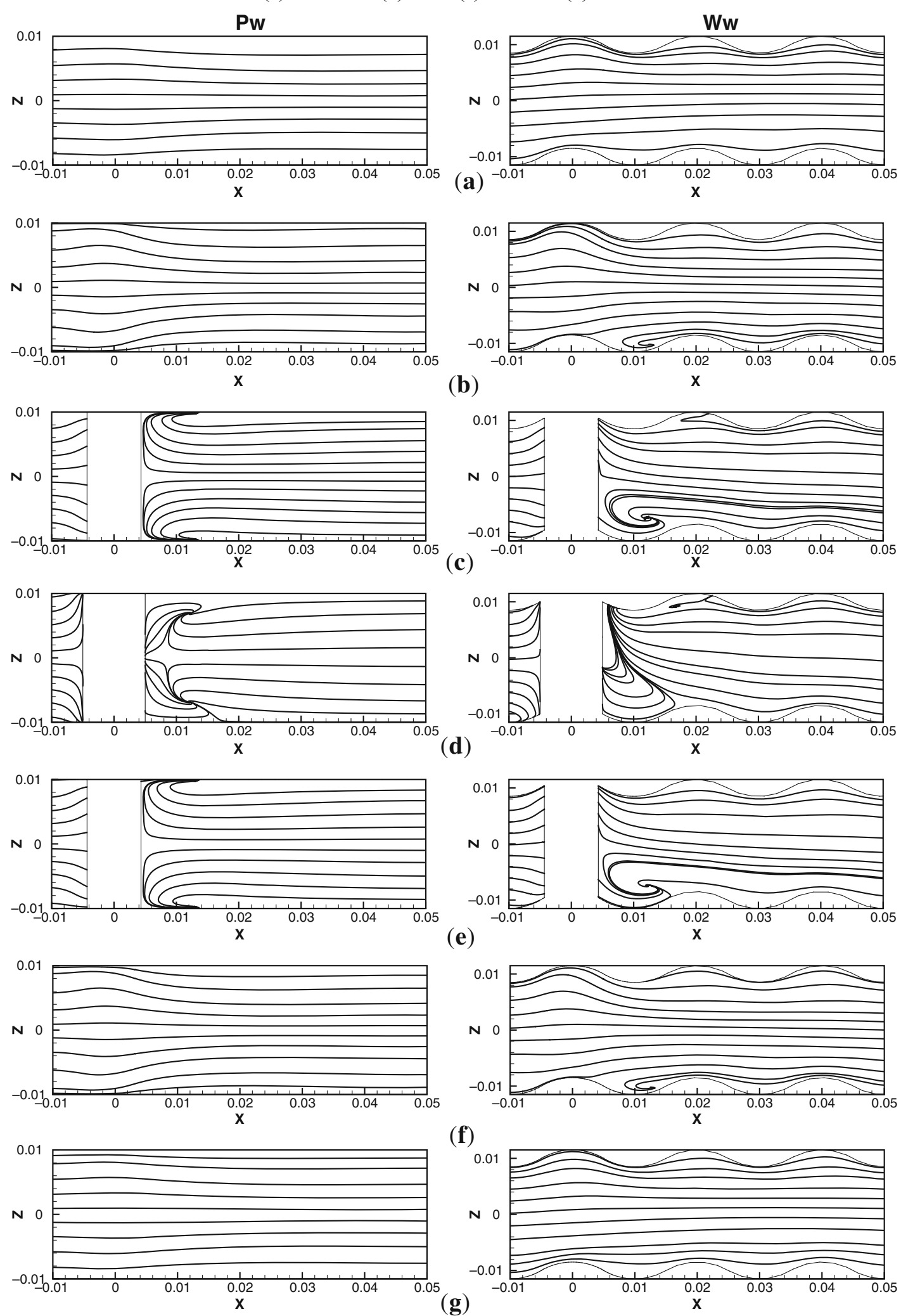

Figure 8. Streamline plots at various $y$-planes for $\operatorname{Re}=20$. (a) $y=0.75 \mathrm{D}$, (b) $y=0.5 \mathrm{D}$, (c) $y=0.25 \mathrm{D},(\mathbf{d}) y=0$, (e) $y=-0.25 \mathrm{D}$, (f) $y=-0.5 \mathrm{D}$ and (g) $y=-0.75 \mathrm{D}$. 
(a) $x=2 D(b) x=3 D(c) x=4 D(d) x=5 D$
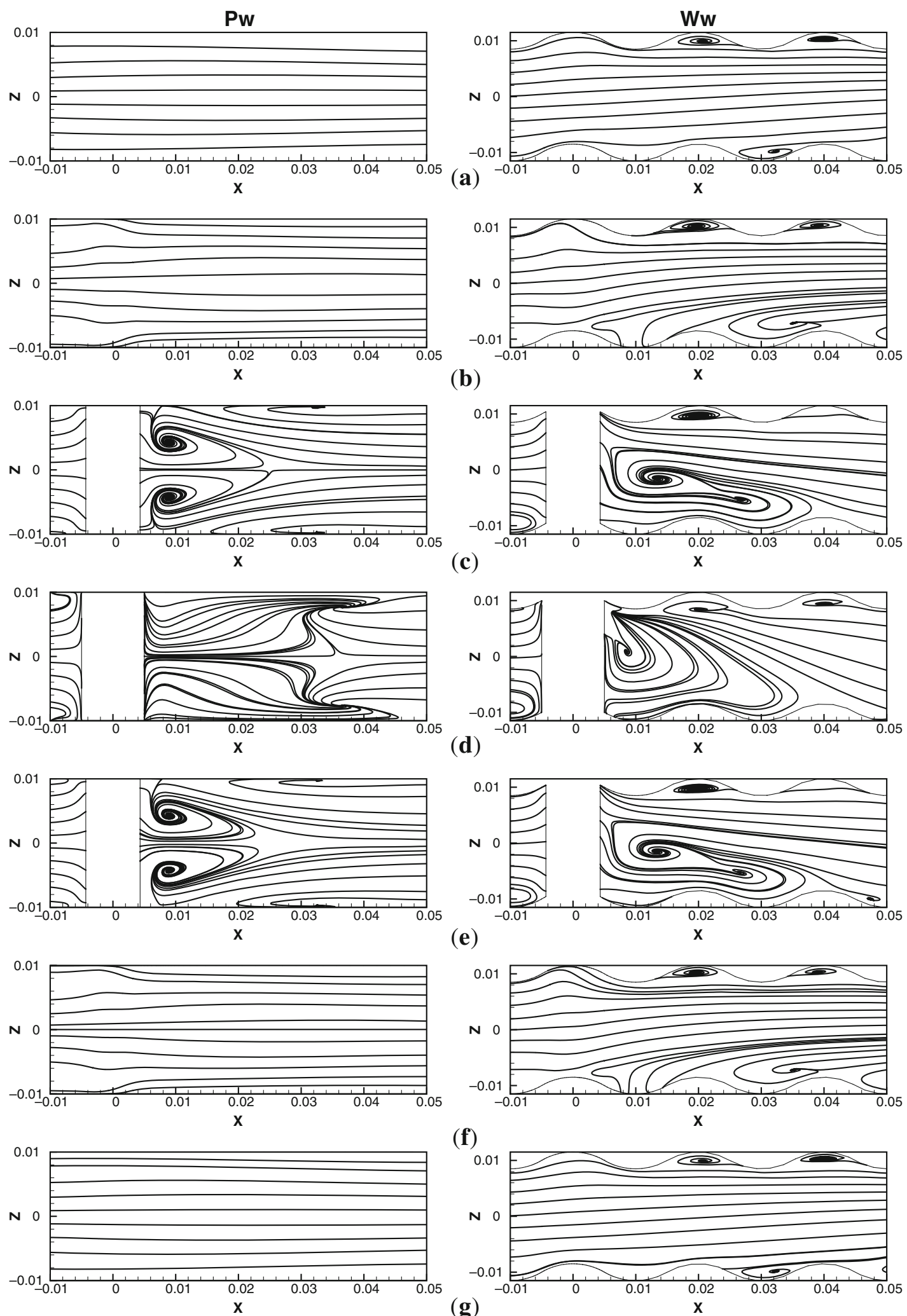

(f)

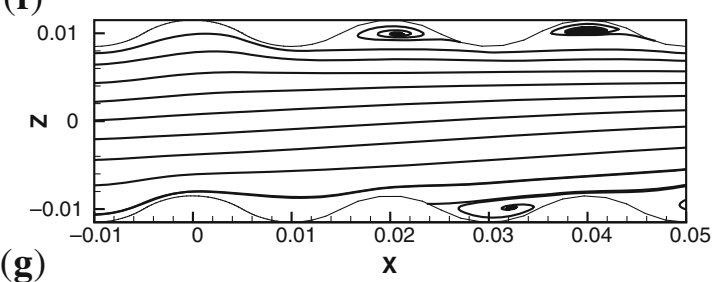

Figure 9. Streamline plots at various $y$-planes for $\operatorname{Re}=100$. (a) $y=0.75 \mathrm{D}$, (b) $y=0.5 \mathrm{D}$, (c) $y=0.25 \mathrm{D}$, (d) $y=0$, (e) $y=-0.25 \mathrm{D}$, (f) $y=-0.5 \mathrm{D}$ and (g) $y=-0.75 \mathrm{D}$. 

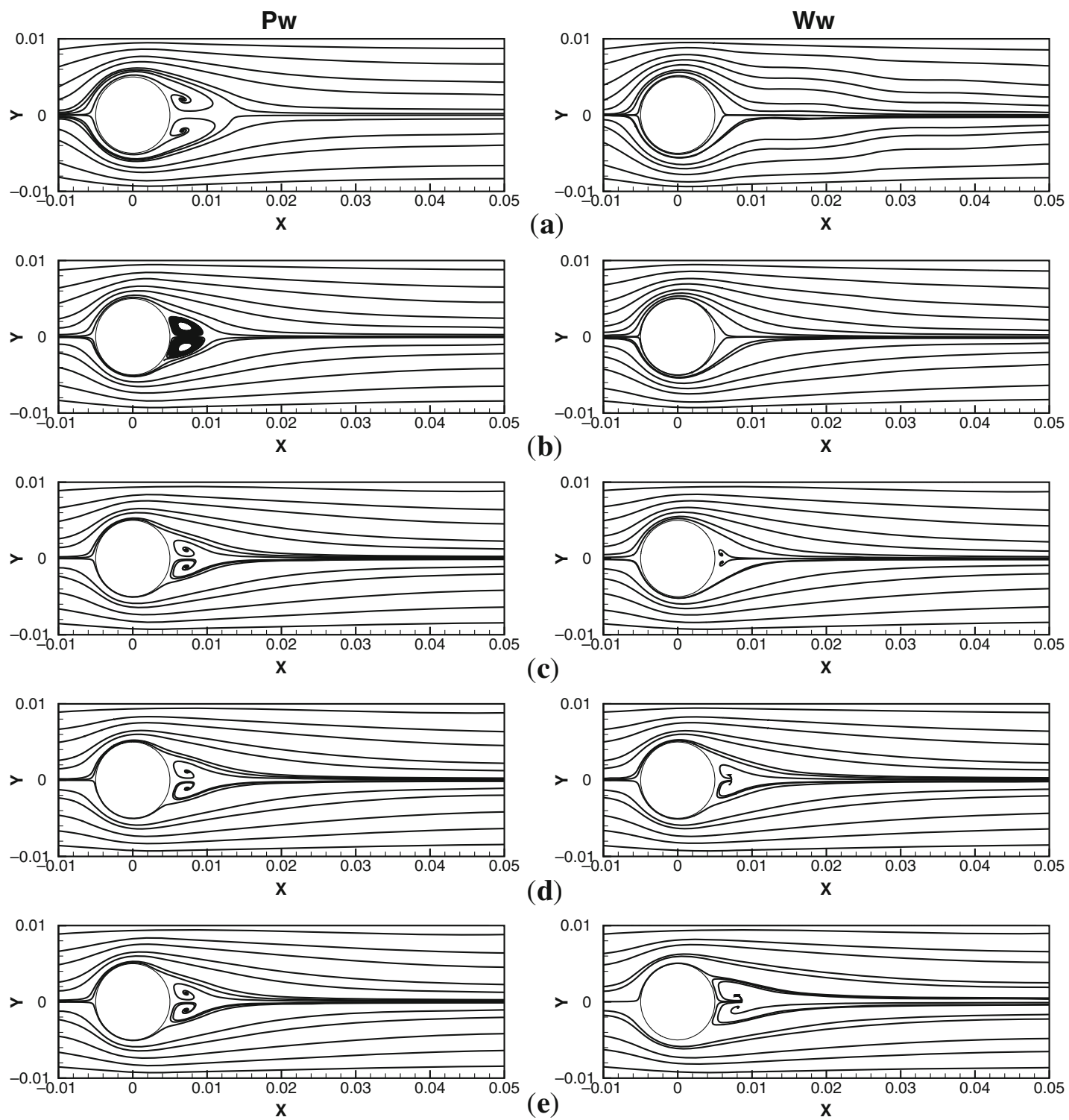

(d)

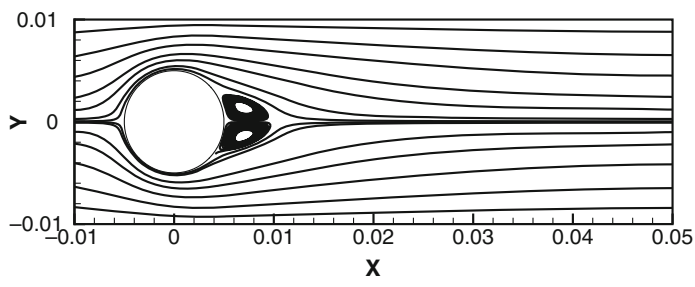

(e)
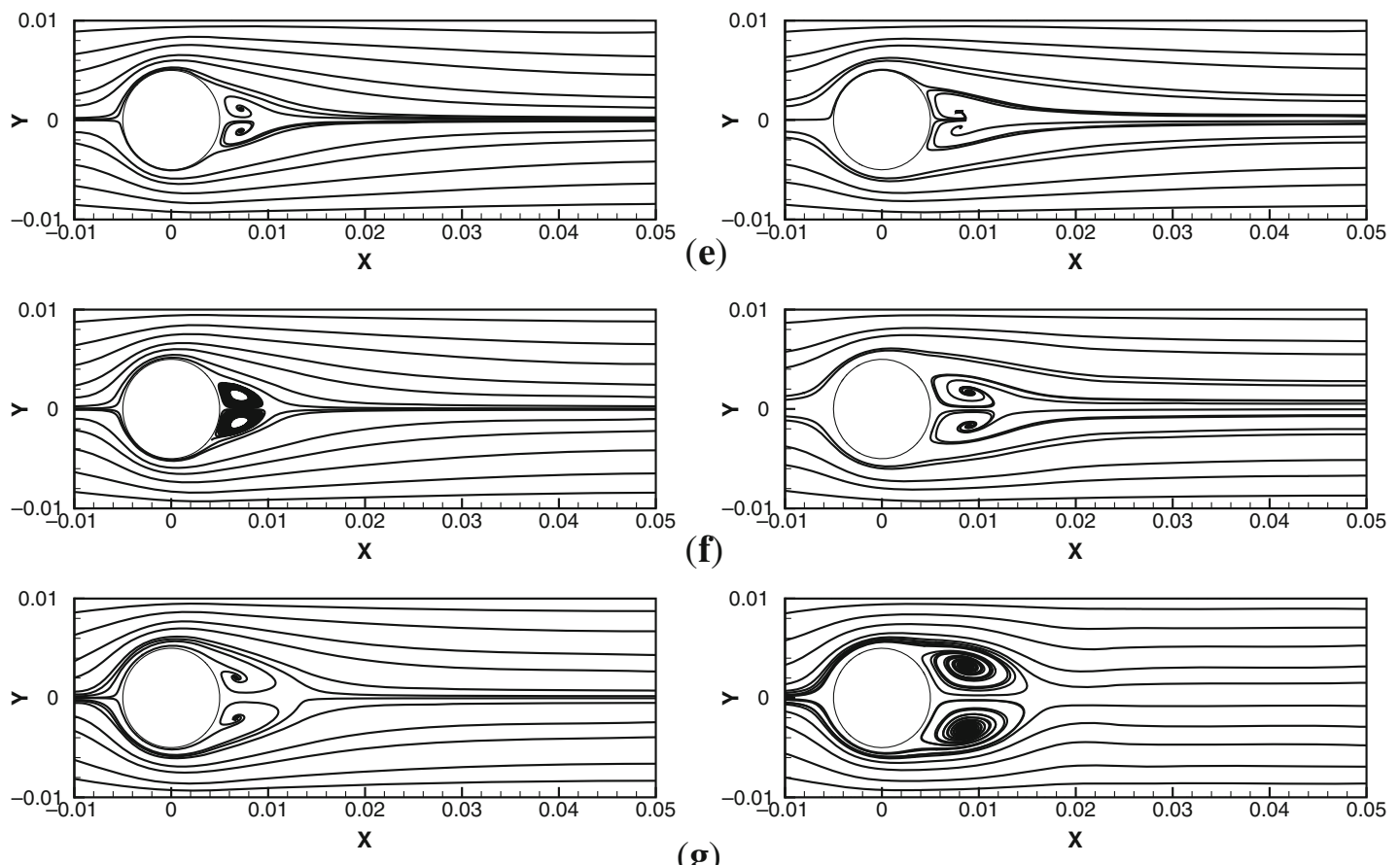

$(\mathbf{g})$

Figure 10. Streamline plots at various z-planes for $\operatorname{Re}=20$. (a) $z=0.75 \mathrm{D},(\mathbf{b}) z=0.5 \mathrm{D},(\mathbf{c}) z=0.25 \mathrm{D},(\mathbf{d}) z=0,(\mathbf{e}) z=-0.25 \mathrm{D}$, (f) $z=-0.5 \mathrm{D}$ and $(\mathbf{g}) z=-0.75 \mathrm{D}$. 

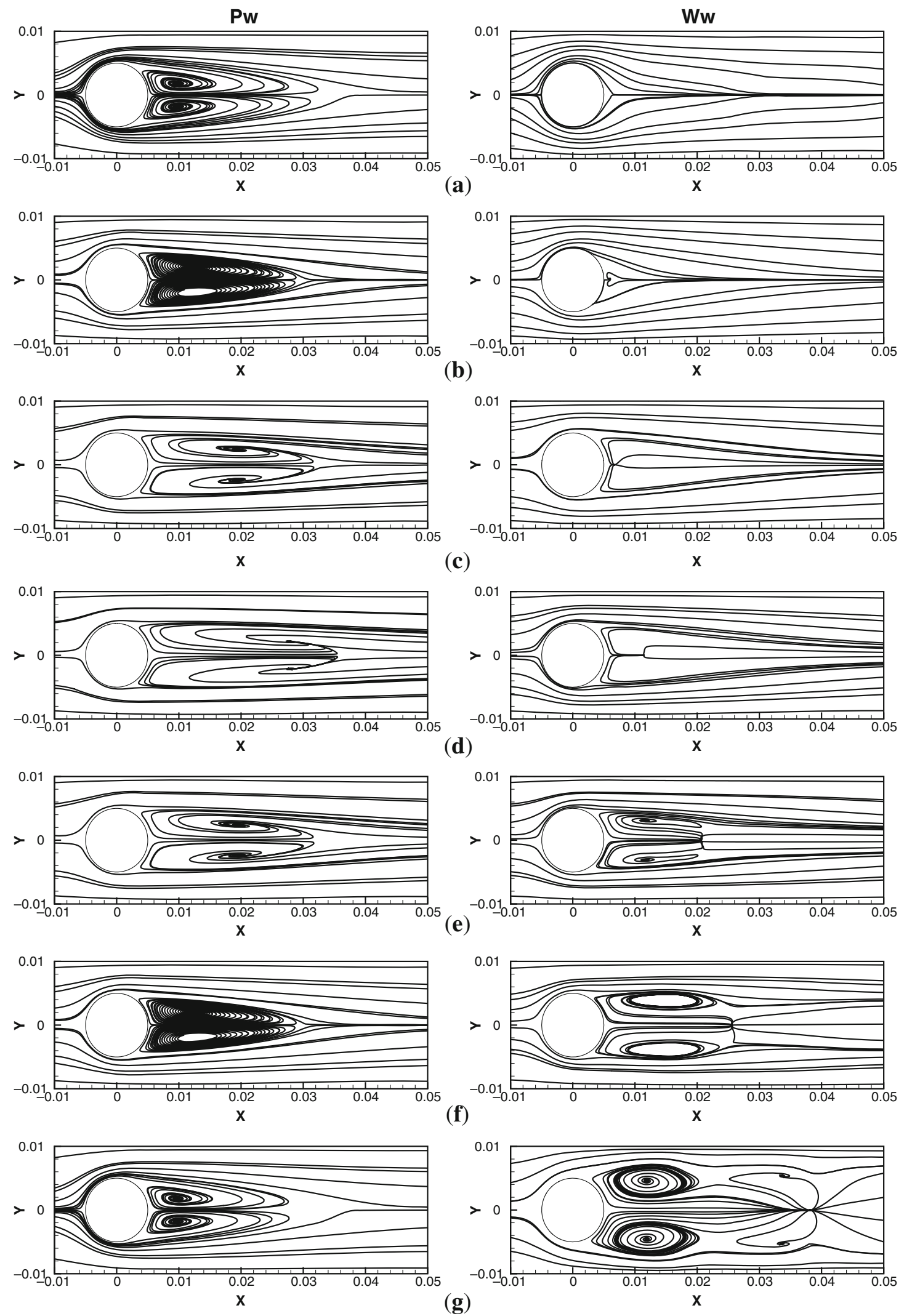

Figure 11. Streamline plots at various $z$-planes for $\operatorname{Re}=100$. (a) $z=0.75 \mathrm{D},(\mathbf{b}) z=0.5 \mathrm{D},(\mathbf{c}) z=0.25 \mathrm{D},(\mathbf{d}) z=0,(\mathbf{e}) z=-0.25 \mathrm{D}$, (f) $z=-0.5 \mathrm{D}$ and $(\mathrm{g}) z=-0.75 \mathrm{D}$. 


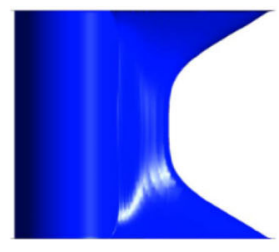

(a) Plain wall, $\operatorname{Re}=20$

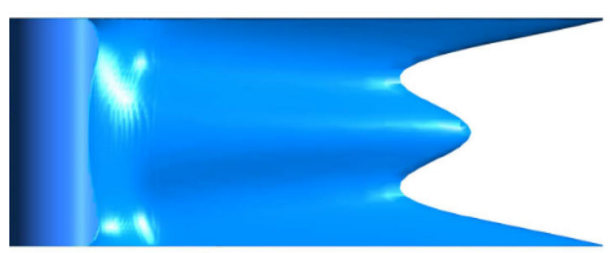

(c) Plain wall, $\mathrm{Re}=100$

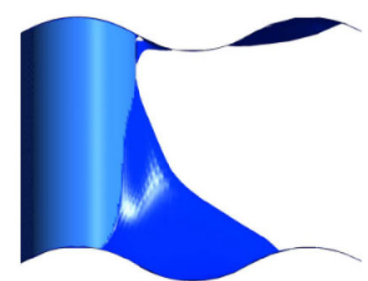

(b) Wavy wall, $\mathrm{Re}=20$

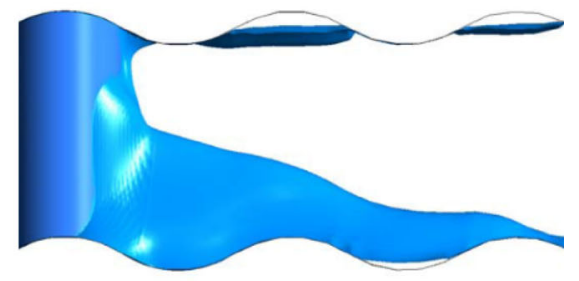

(d) Wavy wall, $\mathrm{Re}=100$

Figure 12. Iso-surface of $u_{x}=0$. (a) Plain wall, $\mathrm{Re}=20$. (b) Wavy wall, $\mathrm{Re}=20$. (c) Plain wall, $\mathrm{Re}=100$. (d) Wavy wall, $\operatorname{Re}=100$.

comparison between plain- and wavy-wall confinements. From these results, it is clearly identified that the wake is strongly affected by the nature of the confining walls.

Within the confined flow domain, the increase in Reynolds number leads to increase in recirculation length, $\mathrm{L}_{\mathrm{r}}$. This nature of variation is similar to the results reported by Rehimi et al [1]. This increase in recirculation length is obviously due to the inertial effect in the wake region and advancement of adverse pressure gradient towards front stagnation point. It is also clear from figure $13 \mathrm{c}$ that the separation angle $\theta_{s}$ decreases with increasing of Reynolds number; the same trend is also observed from Norberg [9], Rajani et al [17], Wu et al [18] and Qu et al [19]. The normalised recirculation length $\left(\mathrm{L}_{\mathrm{r}} / \mathrm{D}\right)$ in an unconfined cylinder at Reynolds number 100 is 1.42 [19]. For the same Reynolds number, the increased recirculation length $\left(\mathrm{L}_{\mathrm{r}} /\right.$ $\mathrm{D}=2.247$ ) obtained by Rehimi et al [1] is purely due to the blockage effect (side walls with no-slip), which stabilises the flow especially in the downstream that results in increasing size of the wake. For an aspect ratio of 30 or above, the cylinder can be treated as an infinite cylinder with negligible wall effect near the mid-plane. In the present study, although the blockage ratio is $1 / 2$, the increased recirculation length $\left(L_{r} / D=3.076\right)$ is not only due to blockage (symmetry boundary condition), but also due to the effect of boundary layer formation from the top and bottom confining solid walls. The work from Huang et al [11] for various confining wall boundary conditions such as the combination of no-slip and free-slip also reveals the same effect of flow stabilisation in downstream as observed in the present study.

Generally, the separation angle decreases with increasing Reynolds number [9, 17-19]. When blockage effect comes into picture, it is interesting to notice that the confining boundary condition plays a significant role in the flow separation, for increasing blockage effect [20] have achieved increasing separation angle (measured from front stagnation point), whereas Wu et al [18] observed decrease in separation angle. This turns our perspective on the effect of boundary condition on flow separation angle. Free stream velocity at the boundaries leads to suppression and delay of flow separation angle with increasing blockage effect. On the other hand, the presence of boundary layer due to no-slip boundary increases the local flow acceleration near cylinder surface, thus resulting in earlier flow separation. In a three-dimensional flow, the angle of separation is also influenced by confining wall effects (aspect ratio effect). Norberg [9] has reported that the flow separation comes earlier when the aspect ratio decreases for a particular Reynolds number. In the present study, the same effect is observed; that is, at Reynolds number 100 the separation angle is 116.36 , whereas for unconfined cylinder the value reported by Qu et al [19] was 118. At low Reynolds number, the effect of wavy wall on $L_{r}$ is almost the same as plain-wall as shown in figure 13b. This indicates that especially at higher Reynolds number the waviness effect is influenced up to the mid-z-plane and at low Reynolds number the viscous effect near the wall overcomes the inertial effect and the wavy-wall influence up to mid- $z$ plane becomes negligible. The comparison of recirculation length clearly shows that for wavy-wall confinement the effect of Reynolds number on $L_{r}$ is almost negligible as compared to plain-wall confinement. This effect is not true for very low Reynolds number, as the wall effect is not felt at the mid-z-plane. Almost constant $\mathrm{L}_{\mathrm{r}}$ with increasing Reynolds number for wavy-wall confined flow is due to the redirected flow towards the main stream from top wall. On the other hand, it is noticed that the presence of waviness also results in increasing the drag, which is due to the increase of local acceleration and fluid shearing near the cylinder surface. Figure 13a shows higher mean drag coefficient with wavy-wall confinement as compared to plain- 


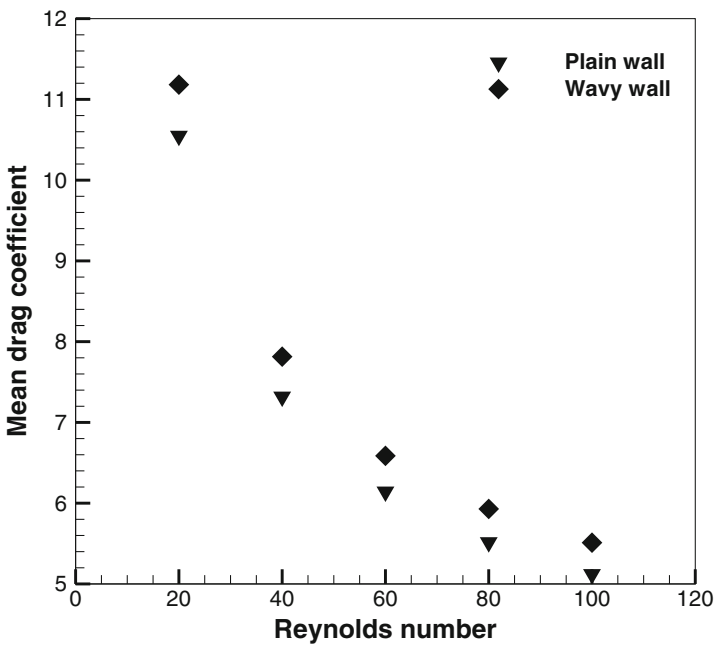

(a)

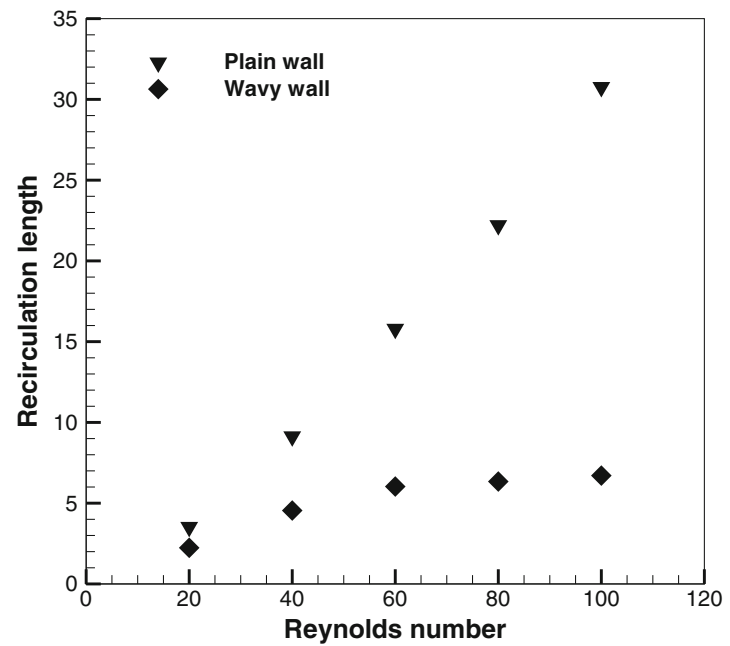

(b)

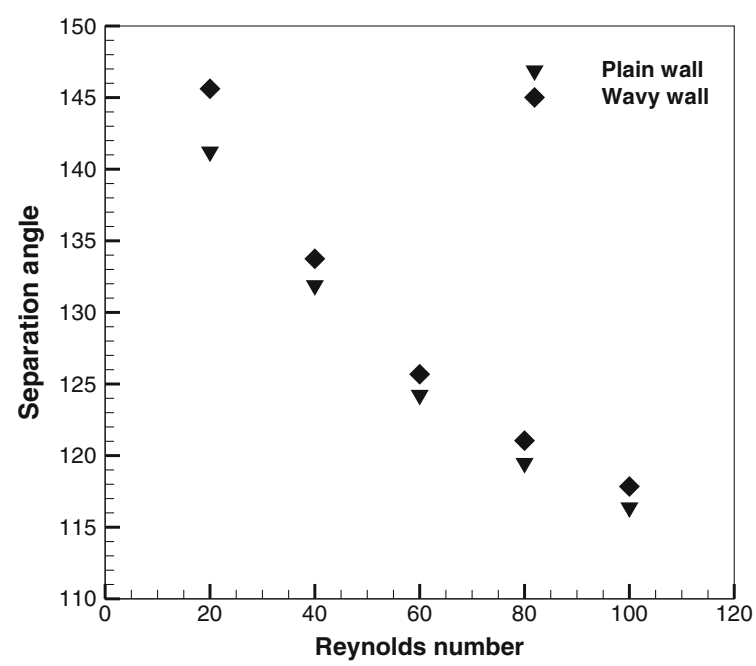

(c)

Figure 13. Effect of plain and wavy walls on flow characteristics. (a) Mean drag coefficient $\left(\mathrm{C}_{\mathrm{D}}\right)$. (b) Recirculation length ( $\left.\mathrm{L}_{\mathrm{r}}\right)$. (c) Separation angle $\left(\theta_{s}\right)$.

wall confinement for all the Reynolds number considered in the present study.

The three-dimensional behaviour of the flow field is significant as discussed in the previous sections, therefore, the $x, y$ and $z$ component wall shear stresses at mid-z-plane for both plain and wavy walls are separately shown in figure 14a to $\mathrm{c}$ for Reynolds number 20 and 100. In the overall picture, it is observed that the $x$-component shear stress along the main flow direction contributes more to the total shear. The $y$-component shear stress is more only near to the front stagnation point where the flow gets redirected along $y$ direction. The zero $x$-component wall shear stress indicates the flow separation, as the Reynolds number increases the flow separation and moves towards the front stagnation point due to the advancement of adverse pressure gradient. For any particular Reynolds number, the separation angle gets delayed in the presence of wavy-wall confinement as compared with plain-wall confinement. It is also noticed that at the flow separation point the $y$-component shear stress also equals zero. The $z$-component shear stress is zero with plain-wall confinement (figure 14c) for all Reynolds numbers considered, which indicates that there is no flow crossing mid $z$-plane. But in the presence of waviness, $z$-component shear stress is experienced by the cylinder up to the mid-plane; especially at high Reynolds number, the significance is more. This effect is due to the waviness in the confining walls.

Separation angle along various $z$-planes for both plain and wavy walls at $\mathrm{Re}=100$ is shown in figure 15 . For plain-wall confinement, the separation angle is a minimum at $z=0$ plane and keeps increasing along both sides of $z$ direction except near to the boundary. This phenomenon results in the reduction of recirculation length as shown in figures 12 and 16, except near the end walls, where the 


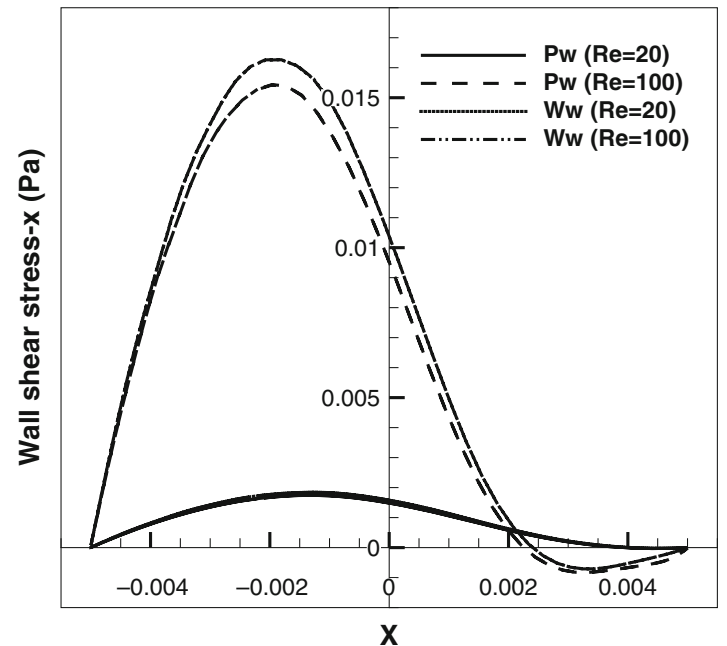

(a)

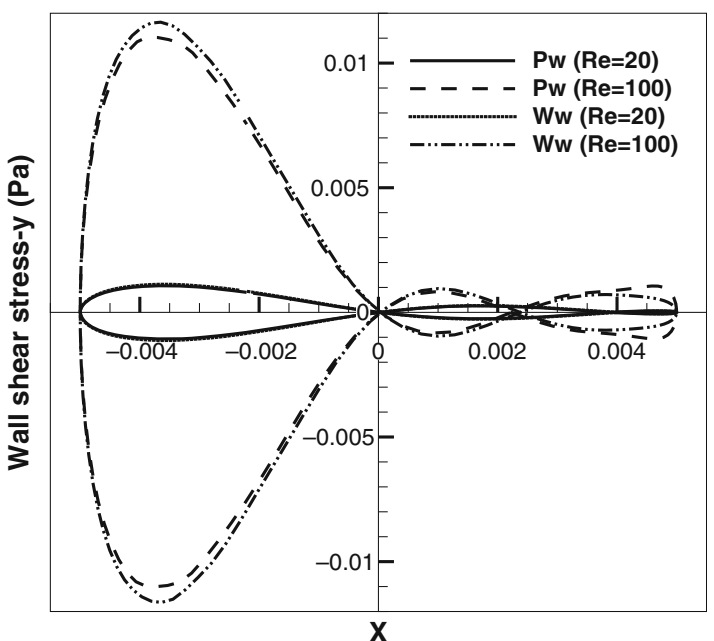

(b)

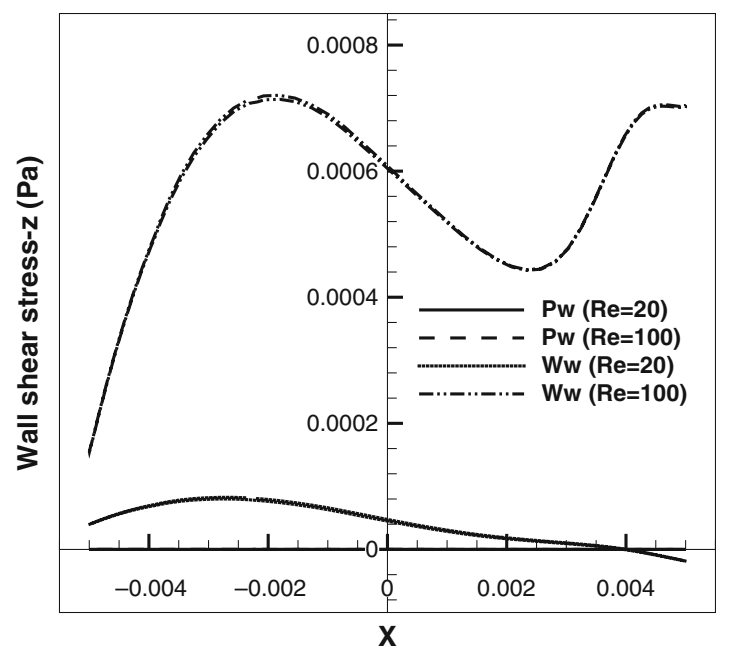

(c)

Figure 14. Individual components of wall shear stress at $z / D=0$. (a) $\tau_{\mathrm{x} .}$ (b) $\tau_{\mathrm{y} .}$ (c) $\tau_{\mathrm{z}}$.

recirculation length increases irrespective of the flow separation angle. It is also observed that for plain wall, the separation angle is symmetric along the axis of the cylinder, whereas in the case of wavy wall there is no flow separation beyond $z / D \approx 0.5$ where flow is attached with cylinder surface due to local acceleration towards downward direction, this behavior is observed from streamline plots as shown in figure 9. At the same time, the wake size increases near the bottom of the cylinder as shown in figure 12. This effect is due to the wall boundary, which leads to local acceleration that stretches the wake bubble.

\subsection{Vortex identification}

Vortices have two components, namely, swirling motion (vorticity) and pure shearing motion (strain-rate), and these two components are of comparable magnitude for vortices generated in wall-confined flows. Therefore, it is essential to distinguish between the two components that contribute to the vortex magnitude. In a flow region, $Q$-criterion due to Hunt et al [21] is one of the methods used to identify the excess magnitude of vorticity relative to strain rate. Chakraborty et al [22] and Kolar [23] have also reported the significance of $Q$-criterion and the procedure to compute the same in an incompressible flow. The characteristics equation for the second-order velocity gradient tensor $\nabla \mathbf{u}$ can be expressed as $\lambda^{3}+P \lambda^{2}+Q \lambda+R=0$, where $Q$ is the second invariant of $\nabla \mathbf{u}$. The invariant $Q$ can be written as $Q=\frac{1}{2}\left([\operatorname{tr}(\nabla \mathbf{u})]^{2}-\operatorname{tr}(\nabla \mathbf{u})^{2}\right)$, where $\operatorname{tr}(\nabla \mathbf{u})=\nabla \cdot \mathbf{u}$ and for an incompressible flow $\nabla \cdot \mathbf{u}=0$. In general, the value $\sqrt{\operatorname{tr}\left[\mathbf{T T}^{T}\right]}$ is nothing but the absolute tensor value (norm) 


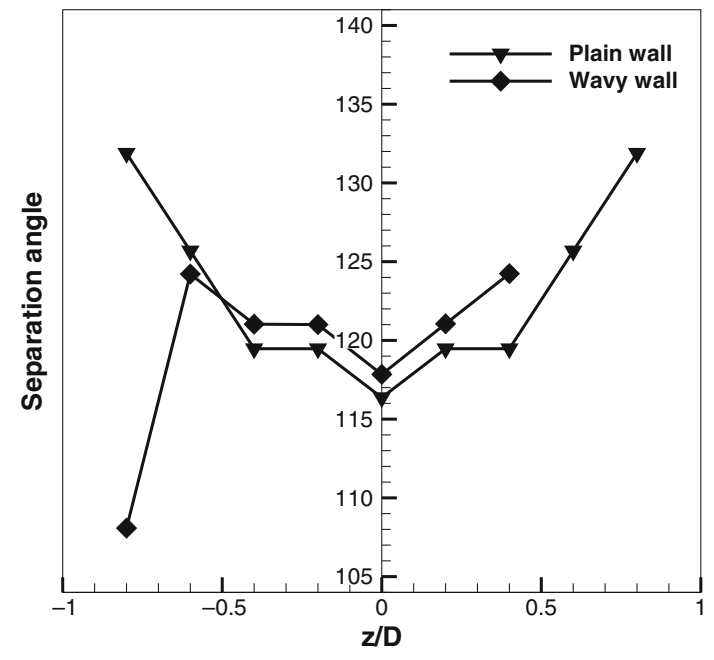

Figure 15. Separation angle along $z$-planes for plain wall and wavy wall at $\mathrm{Re}=100$.

$\|\mathbf{T}\|$, where $\mathbf{T}$ is any tensor. Further, the velocity gradient tensor, $\nabla \mathbf{u}$, can be decomposed as $\nabla \mathbf{u}=\mathbf{S}+\boldsymbol{\Omega}$, where $\mathbf{S}$ is the symmetric strain-rate component and $\boldsymbol{\Omega}$ is the antisymmetric vorticity component. Thus, the $Q$-criterion for an incompressible flow is $Q=\frac{1}{2}\left(\|\mathbf{\Omega}\|^{2}-\|\mathbf{S}\|^{2}\right)$. As described earlier, for $(Q>0)$ the region covers the vortices where the magnitude of vorticity is greater than the magnitude of strain rate in all directions. The additional requirement for using $Q$-criterion is that pressure in the vortex region should be lower than the ambient pressure. Higher value of $Q$ indicates higher vortex strength and correspondingly reduction in vortex region and vice versa.

The $\lambda_{2}$-criterion due to Jeong and Hussain [24] is used to ensure the existence of a pressure minimum inside the vortex region. The vorticity transport equation and strain-

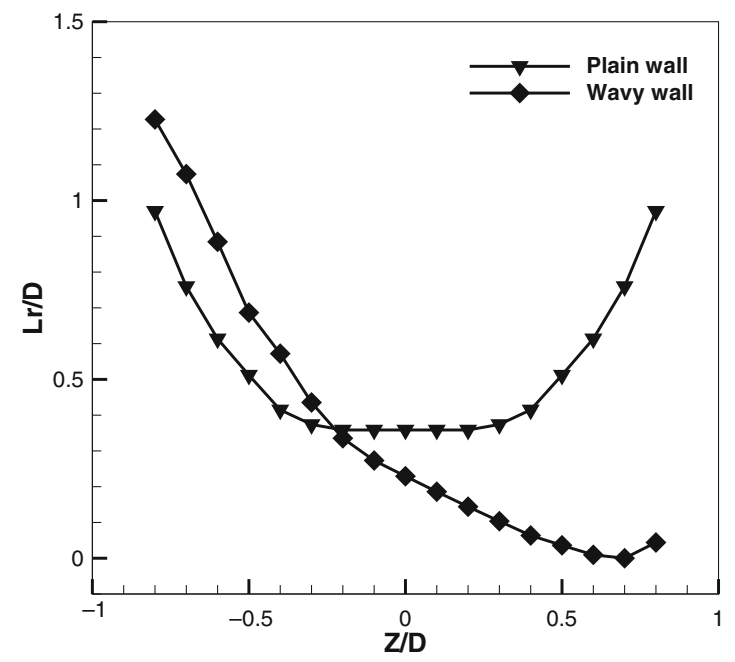

(a) rate transport equations are derived by taking the gradient of N-S equations and decomposing the resulting acceleration gradient into symmetric and anti-symmetric components. The strain-rate transport equation consists of an unsteady term, viscous contributions, $S^{2}+\Omega^{2}$ term and pressure Hessian. To calculate the excess magnitude of vorticity relative to strain-rate, unsteady and viscous terms are removed as reported by Jeong and Hussain [24]. Further, if the eigen values are ordered as $\lambda_{1} \geq \lambda_{2} \geq \lambda_{3}$, to have two negative eigen values of $S^{2}+\Omega^{2}$, the requirement is that the second eigen value, $\lambda_{2}<0$.

The present study captures the complex three-dimensional flow characteristics due to cylinder and wall interaction. Formation of vortices near troughs of the wavy wall close to the cylinder is clearly observed from the streamline plots where the vortical structures are not strictly lined up with a coordinate axis, the vortices are not captured by the streamline plots in good order. Moreover, the observed vortices are found in shear layer close to the wall where velocity gradients are high, straining and pure shearing motion in the region do not contribute to actual swirling motion, $g$, of a vortex and the study of iso-vorticity surface will be misleading. Therefore, $Q$-criterion and $\lambda_{2}$-criterion as discussed earlier are used for vortex identification, which effectively distinguish vorticity magnitude and strain rate. Figures 17 and 18 show the iso-surface of $Q$ - and $\lambda_{2}-$ criteria for both plain- and wavy-wall confinement at Reynolds numbers 20 and 100 . The values for iso-surface are taken as $0.1 \%$ of $Q_{\max }$ and $0.1 \%$ of $\lambda_{2} \min$.

Apparently, the vortex structure obtained using $Q$ method is very similar to those obtained from $\lambda_{2}$ method. Zhou et al [25] have also reported that various vortex identification methods capture near-wall vortical structure almost in a similar fashion. It is also observed that the vortex strength is higher at low recirculation region and

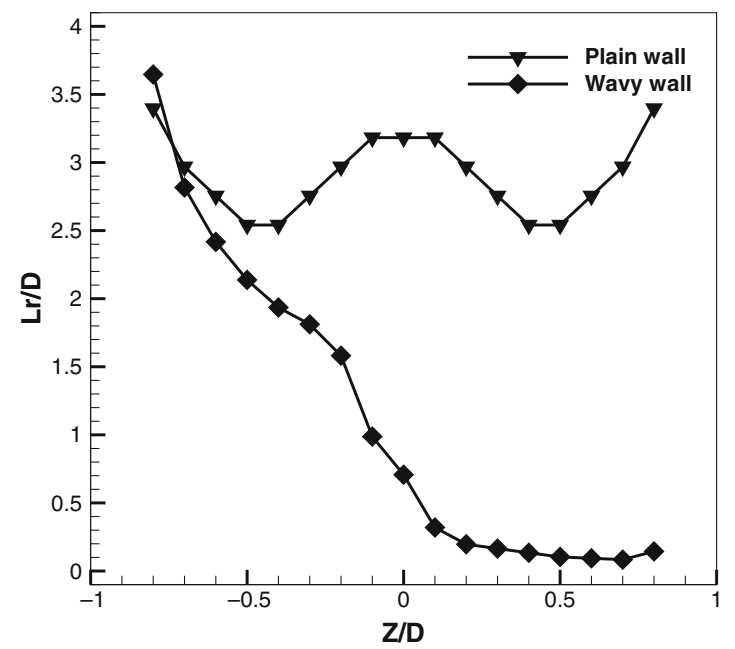

(b)

Figure 16. Recirculation length along $z$-planes for plain wall and wavy wall at (a) $\operatorname{Re}=20$ and $(\mathbf{b}) \operatorname{Re}=100$. 


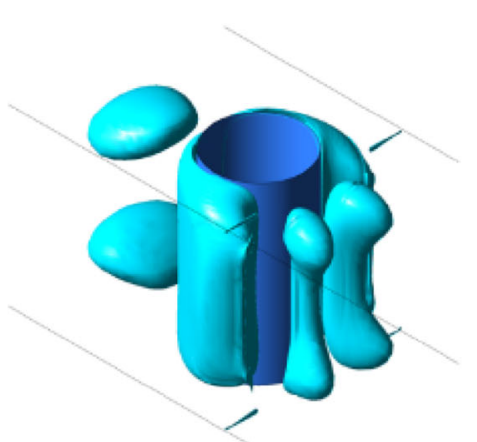

$\mathrm{Re}=20\left(\mathrm{Q}_{\max }=385.6\right)$

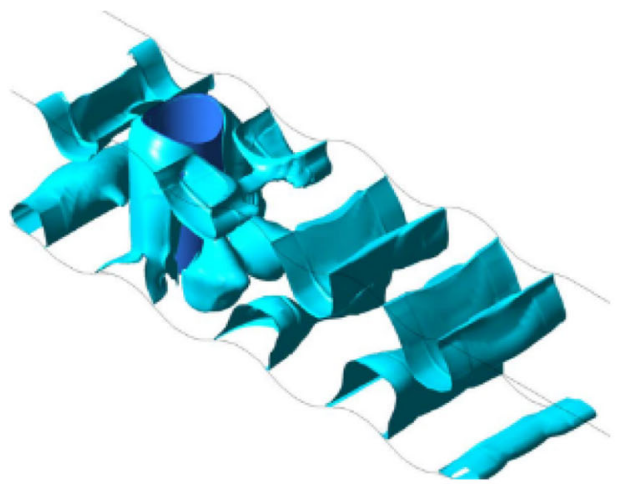

$\mathrm{Re}=20\left(\mathrm{Q}_{\max }=423.8\right)$
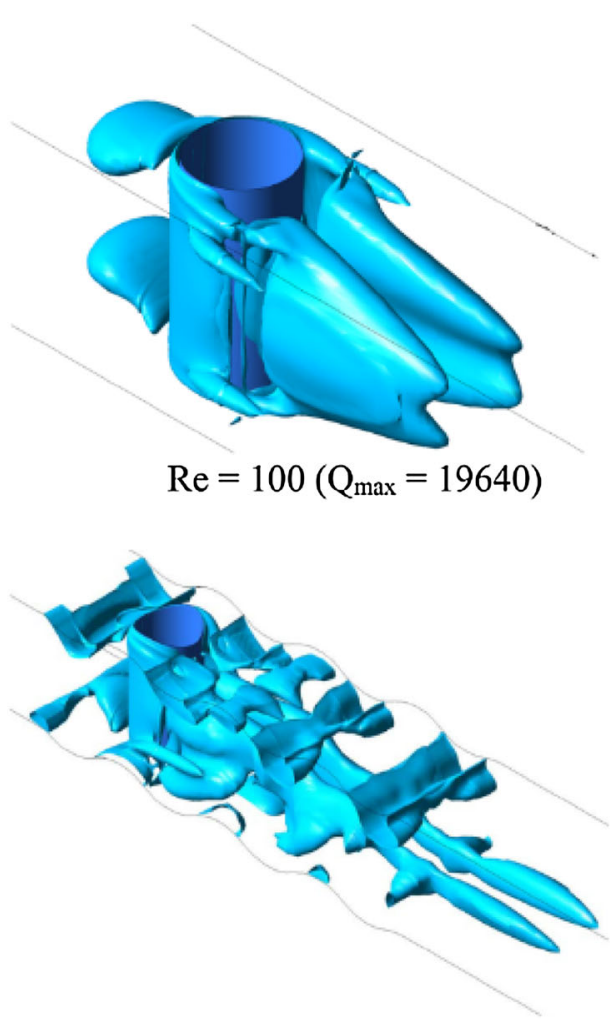

$\operatorname{Re}=100\left(Q_{\max }=21460\right)$

Figure 17. Iso-surface of $Q\left(Q=0.1 \% Q_{\max }\right)$.

vice versa. Stream-wise vortices behind the cylinder are clearly observed from figures 17 and 18; the vortices grow further at higher Reynolds number. With plain-wall confinement, the well-defined vortex growth is observed near the centreline of the domain. With plain-wall confinement, due to the symmetrical top and bottom wall confinement, stream-wise vortices with axes almost oriented parallel to $x$-direction are observed. With wavy-wall confinement, the presence of troughs and crests leads to vortices with axes almost oriented parallel to $y$ direction. Therefore, wavywall confinement leads to generation of swirling vortices near crest and trough and these vortices are responsible for breaking the growth of stream-wise vortices behind the cylinder. Two local swirling vortices are observed at low Reynolds number $(\operatorname{Re}=20)$, however, with increasing Reynolds number merging of these swirling vortices takes place.

\section{Conclusions}

Three-dimensional numerical simulations of laminar flow past circular cylinder in the Reynolds number range $20 \leq \mathrm{Re} \leq 100$ have been carried out in presence of plain and wavy-wall confinements. The wavy-wall confinement leads to elimination of recirculation near top end of the cylinder, increased drag and formation of $x$-plane vortices. Increase in recirculation size with increasing Reynolds number is observed for both plain- and wavy-wall confinements. However, with wavy-wall confinement for all value of $\mathrm{Re}$, the flow is redirected into the cylinder wake and eliminates flow recirculation near top end of the cylinder. Increased drag with wavy-wall confinement is due to local acceleration of the flow. The effect of confining wavy-walls penetrates up to mid-z-plane of the domain thus increases the flow mixing, which is much less with plainwall confinement.

Even though there is no vortex shedding in presence of highly confined wavy wall, it leads to complex three-dimensional flow behaviour in the cylinder wake. The generation of $x$-plane vortices in the downstream is significant at higher Reynolds number $(\operatorname{Re}=100)$, and formation of $x$ plane vortices in the trough region of the wavy wall results in more pressure drop. Such vortices are present in the plain-wall-confined flow domain up to and even beyond the downstream length of 3.5D. On the other hand, for wavywall confinement, these vortices are present either near top wall or bottom wall depending on location of trough region of the wavy wall. It is well known that the flow is twodimensional for flow past unconfined circular cylinder in the Reynolds number range $20 \leq \mathrm{Re} \leq 100$, however, from the present study, the formation of $y$-plane $(y= \pm$ 

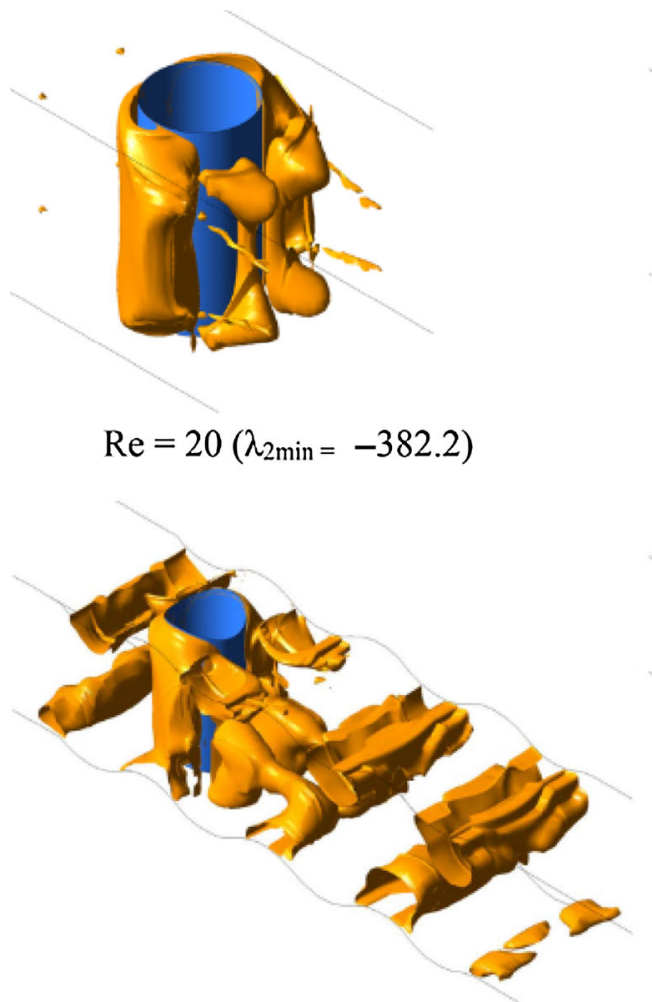

$\operatorname{Re}=20\left(\lambda_{2 \min }=-423.5\right)$

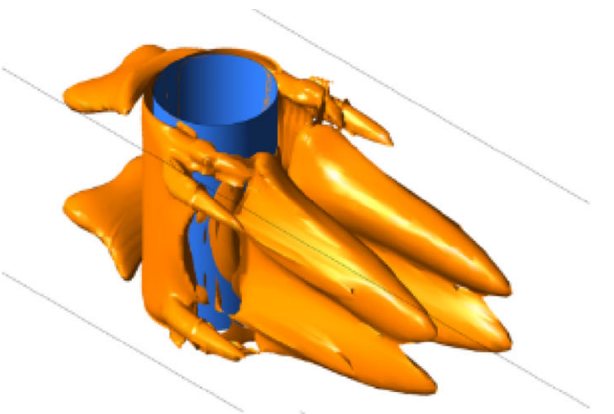

$\operatorname{Re}=100\left(\lambda_{2 \min }=-18640\right)$

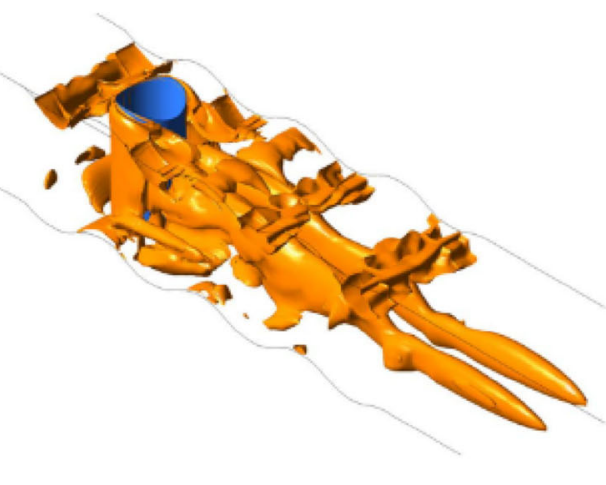

$\operatorname{Re}=100\left(\lambda_{2 \min }=-20420\right)$

Figure 18. Iso-surface of $\lambda_{2}\left(\lambda_{2}=0.1 \% \lambda_{2 \mathrm{~min}}\right)$.

$0.25 \mathrm{D})$ vortices in the cylinder wake clearly indicates the three-dimensional flow behaviour due to channel confinement. The study will be more interesting for further increase in Reynolds number where the interactive effect of wavy wall and vortices shed from the cylinder will be highly significant.

\section{Nomenclature}

A waviness amplitude of the confining wall

$A P \quad$ projected area of the cylinder

$C_{D} \quad$ drag coefficient

$D \quad$ diameter of the cylinder

$H \quad$ distance between the symmetric front and rear sides (along $y$-direction)

$K \quad$ wave number

$L \quad$ distance between the top and bottom confining walls (along $z$-direction)

$L_{d} \quad$ downstream length of domain

$L_{r} \quad$ recirculation Length (measured between rear stagnation point and the point where $x$-velocity reaches zero in the centerline of downstream)

$n \quad$ number of grids

$P \quad$ pressure

$\mathrm{Pw} \quad$ plain-wall

$R \quad$ radius of the cylinder

Re Reynolds number
$\mathrm{S} \quad$ rate-of-strain tensor

$U \quad x$-component velocity at the inlet

$\mathrm{U} \quad$ velocity vector

$u, v, w \quad x-, y$ - and $z$-component velocities, respectively

$x, y, z \quad$ coordinate axes

Ww wavy wall

$\mathrm{C}_{\mathrm{D}} \quad$ mean Drag co-efficient $=\left(F_{D}\right) /\left(1 / 2 \rho U_{\text {avg }}^{2} A P\right)$

\section{Greek symbols}

$\alpha \quad$ aspect ratio

$\beta \quad$ blockage ratio

$\lambda$ wave length

$\mu \quad$ dynamic viscosity

$\rho \quad$ density

$\theta_{s} \quad$ separation angle measured from front stagnation point

$\Omega \quad$ vorticity tensor

$\theta_{\tau \max }$ separation angle at maximum wall shear stress

\section{Subscripts}

avg average

$\max$ maximum

$\min$ minimum 


\section{References}

[1] Rehimi F, Aloui F, Ben Nasrallah S, Doubliez L and Legrand J 2008 Experimental investigation of a confined flow downstream of a circular cylinder centered between two parallel walls. J. Fluids Struct. 24: 855-882

[2] Williamson C H K 1996 Three-dimensional wake transition. J. Fluid Mech. 328: 345-407

[3] Williamson C H K 1996 Vortex dynamics in the cylinder wake. Annu. Rev. Fluid Mech. 28: 477-539

[4] Shaligram T, Pratish PP, Jayavel S and Biswas G 2006 Flow field characteristics near first transition for flow past a circular tube confined in a narrow channel. In: Proceedings of the 3rd International and 33rd National Conference on Fluid Mechanics and Fluid Power. 1132, IIT Mumbai, India

[5] Deepakkumar R, Jayavel S and Shaligram T 2016 Computational study of fluid flow characteristics past circular cylinder due to confining walls with local waviness. Journal of Physics: Conference Series, volume 759, number $1 / 012083$

[6] Sahin M, Robert G and Owens A 2004 Numerical investigation of wall effects up to high blockage ratios on twodimensional flow past a confined circular cylinder. Phys. Fluids. 16: 1305-1320

[7] Kanaris N, Grigoriadis D and Kassinos S 2011 Three dimensional flow around a circular cylinder confined in a plane channel. Phys. Fluids. 23: 064-106

[8] Zovatto L and Pedrizzetti G 2001 Flow about a circular cylinder between parallel walls. J. Fluid Mech. 440: 1-25

[9] Norberg C 1994 An experimental investigation of the flow around a circular cylinder: influence of aspect ratio. J. Fluid Mech. 258: 287-316

[10] Detlev G and Eckelmann H 1982 Influence of end plates and free ends on the shedding frequency of circular cylinders. $J$. Fluid Mech. 122: 109-121

[11] Zhiyong H, Andersson H I and Cui W 2011 End-wall effects on vortex shedding in planar shear flow over a circular cylinder. Comp. Fluids. 42: 102-107

[12] Atul S and Eswaran V 2005 Effect of channel confinement on the two-dimensional laminar flow and heat transfer across a square cylinder. Numer. Heat Transfer A 47: 79-107

[13] Bearman P W 1997 Near wake flows behind two and threedimensional bluff bodies. J. Wind Eng. Ind. Aerodyn. 69: $33-54$
[14] Jiehai Z, Kundu J and Manglik R M 2004 Effect of fin waviness and spacing on the lateral vortex structure and laminar heat transfer in wavy-plate-fin cores. Int. J. Heat Mass Transfer 47: 1719-1730

[15] Ribeiro V M, Coelho P M, Pinho F T and Alves M A 2012 Three-dimensional effects in laminar flow past a confined cylinder. Chem. Eng. Sci. 84: 155-169

[16] Deepakkumar R and Jayavel S 2014 Effect of under relaxation factor on convergence rate of computations for flow past circular cylinder. In: Proceedings of 6th International Conference on Theoretical, Applied, Computational and Experimental Mechanics, Paper No. ICTACEM - 123, IIT Kharagpur, India

[17] Rajani B N, Kandasamy A and Sekhar M 2009 Numerical simulation of laminar flow past a circular cylinder. Appl. Math. Modeling 33: 1228-1247

[18] Wu M H, Wen C Y, Yen R H, Weng M C and Wang A B 2004 Experimental and numerical study of the separation angle for flow around a circular cylinder at low Reynolds number. J. Fluid Mech. 515: 233-260

[19] Qu L, Christofer N, Lars D, Shia-Hui P and Fujun W 2013 Quantitative numerical analysis of flow past a circular cylinder at Reynolds number between 50 and 200. J. Fluids Struct. 39: 347-370

[20] Chakraborty J, Nishith V and Chhabra R P 2004 Wall effects in flow past a circular cylinder in a plane channel: a numerical study. J. Chem. Eng. Process. 43: 1529-1537

[21] Hunt J C R, Wray A A and Moin P 1988 Eddies, streams and convergence zones in turbulent flows. In: Proceedings of the Summer Program, Center for Turbulence Research, N8924555

[22] Chakraborty P, Balavhandar S and Ronald J A 2005 On the relationships between local vortex identification schemes. $J$. Fluid Mech. 535: 189-214

[23] Kolar V 2007 Vortex identification: new requirements and limitations. Int. J. Heat Fluid Flow. 28: 638-652

[24] Jeong J and Hussain F 1995 On the identification of a vortex. J. Fluid Mech. 285: 69-94

[25] Zhou J, Adrian R J, Balachandar S and Kendall T M 1999 Mechanisms for generating coherent packets of hairpin vortices in channel flow. J. Fluid Mech. 387: 353-396 